\title{
INVARIANT SOLUTIONS TO THE ORIENTED PLATEAU PROBLEM OF MAXIMAL CODIMENSION
}

\author{
BY \\ DAVID BINDSCHADLER
}

\begin{abstract}
The principal result gives conditions which imply that a solution to the Plateau problem inherits the symmetries of its boundary. Specifically, let $G$ be a compact connected Lie subgroup of $\mathrm{SO}(n)$. Assume the principal orbits have dimension $m$, there are no exceptional orbits and the distribution of $(n-m)$ planes orthogonal to the principal orbits is involutive. We show that if $B$ is a finite sum of oriented principal orbits, then every absolutely area minimizing current with boundary $B$ is invariant.

As a consequence of the methods used, the above Plateau problems are shown to be equivalent to 1-dimensional variational problems in the orbit space. Some results concerning invariant area minimizing currents in Riemannian manifolds are also obtained.
\end{abstract}

1. Introduction. The structure of solutions to the oriented Plateau problem has been under investigation since their existence was established by Federer and Fleming in 1960 [FF]. One question that has arisen is whether solutions to the Plateau problem inherit the symmetries of their boundaries. Specifically, if $G \subset$ $\mathrm{O}(n)$ is a compact Lie group acting on $\mathbf{R}^{n}$ and $B_{0}$ is the boundary of an integral current with $B_{0}$ invariant under the action of $G$, then must a solution to the Plateau problem with boundary $B_{0}$ also be invariant? Indeed, does there exist even one invariant solution? Federer has shown that in general there may exist no invariant solution [F1, 5.4.17]. However, Lawson proved that if $G \subset \operatorname{SO}(n)$ and $B_{0}$ is a connected, oriented manifold of dimension $n-2$ lying on the unit sphere $S^{n-1}$, then there exist invariant solutions [L]. Here we show that if $G$ is connected, there are no exceptional orbits, the distribution of planes orthogonal to the principal orbits is involutive, and $B_{0}$ is a finite sum of oriented principal orbits, then every solution to the oriented Plateau problem with boundary $B_{0}$ is invariant. Moreover, the Plateau problem with boundary $B_{0}$ is equivalent to a 1 -dimensional variational problem in the orbit space.

Of central importance to this work is the projecting and lifting of currents in the bundle induced by the group action. If $G \subset \operatorname{SO}(n)$ is as above and $X$ is the set of points which lie on principal orbits (orbits of highest dimension), then $X$ is an open

Received by the editors August 21, 1978.

AMS (MOS) subject classifications (1970). Primary 49F20, 49F22; Secondary 28A70, 53C65, 55F10, $58 \mathrm{~A} 10$.

Key words and phrases. Invariant solutions, area minimizing, mass minimizing, oriented Plateau problem, variational problem, lifting currents, projecting currents, involutive distribution, group action, orbit space.

'This research was partially supported by Wayne State Faculty Research Award number 167-1588. 
dense subset of $\mathbf{R}^{n}$ and admits the structure of a fibre bundle with base space $X / G$ and fibre diffeomorphic to a principal orbit [BG, II.5.8]. However, currents of compact support in $\mathbf{R}^{n}$ may not have compact support when restricted to $X$. Thus we extend Brothers lifting operator [BJ1, 3.3] to currents with arbitrary support that are representable by integration and establish some of the basic properties of this extension.

In $\S 4$, we establish a useful characterization of currents in $X$ that are lifts (4.2; compare [BJ1, 3.7]) and show that the mass of a current is not increased if the current is projected to $X / G$ then lifted back up to $X$. Indeed, 4.8(2) implies that the mass of a current $T$ of finite mass is strictly decreased by projecting then lifting $T$ unless $T$ is tangent to the orbits of $G$. It is this fact together with the involutivity assumption (which implies that $\partial$ commutes with the projection operator) which implies that any locally flat current with boundary $B_{0}$ that minimizes mass among all locally flat currents with boundary $B_{0}$ is a lift and hence invariant (5.2).

In order to obtain positive results concerning integral currents, we show that the problem of minimizing mass among locally flat currents in $X$ is equivalent to a 1-dimensional variational problem (with real coefficients) in $X / G$. Restricting to compact subsets of $X / G$ we apply [F2, 5.12] from which it follows that solutions to the original problem minimize mass not only among integral currents with boundary $B_{0}$, but also, when restricted to $X$, among locally flat currents with boundary $B_{0}$. The invariance of the original solution then follows from 5.2.

Since most of the techniques are independent of Euclidean space, the following result is also obtained: Let $M$ be a compact orientable Riemannian manifold and let $G$ be a compact connected Lie group of isometries of $M$. Suppose that there is one orbit type and the distribution of planes orthogonal to the orbits is involutive. If $B_{0}$ is a finite union of oriented orbits, then any integral current with boundary $\boldsymbol{B}_{0}$ which minimizes mass among all such currents is invariant.

Most of the results here are from [B]. However, 3.5, 3.6, the stronger versions of 5.2 and 5.7, 5.8, 5.10 and 5.11 are new. It should be noted that [BJ1, 3.6 and 3.7], [BJ2, 3.4] and [L, Theorems 2(c) and 4] have served as inspiration and in some cases models for $3.1,4.2,3.4,5.4$ and 5.5, respectively. I would also like to express my deep appreciation for the helpful suggestions and advice that I have received from John E. Brothers.

2. Notation. We adopt the notation of [F1, pp. 669-671] except for the following additions, changes and restrictions.

The identity map on a set $A$ will be denoted by id $A$.

We will use $T_{x} M$ to denote the tangent space to a manifold $M$ at the point $x \in M$, and $T M$ will be the tangent bundle of $M$.

If $M$ and $N$ are $C^{\infty}$ manifolds and $f: N \rightarrow M$ is $C^{\infty}$, then

$$
f_{\sharp}: T N \rightarrow T M
$$

is the (intrinsic) differential of $f$. In case $f$ is $1-1$ and $\xi$ is a vectorfield on $N$, we define $f_{\sharp} \xi$ by 


$$
f_{\sharp} \xi(x)=f_{\sharp}\left(\xi\left(f^{-1}(x)\right)\right)
$$

for $x \in \operatorname{im} f$.

Let $M$ be an oriented $n$-dimensional Riemannian manifold with metric $b$ and unit orienting $n$-vectorfield $\vec{M}$. For each $0 \leqslant k \leqslant n$ let $\gamma_{k}: \wedge_{k} T M \rightarrow \wedge^{k} T M$ be the isomorphism characterized by

$$
\left\langle\alpha, \gamma_{k}(\beta)\right\rangle=b(\alpha, \beta)
$$

for $\alpha, \beta \in \wedge_{k} T_{x} M$ and $x \in M$. The Hodge duality operators are the maps

$$
*: \wedge_{k} T M \rightarrow \wedge_{n-k} T M \text { and } *: \wedge^{k} T M \rightarrow \wedge^{n-k} T M
$$

defined by the formulae

$$
* v=\vec{M}(x)\left\llcorner\gamma_{k}(v) \text { and } * \omega=\gamma_{n-k}(\vec{M}(x)\llcorner\omega)\right.
$$

for $x \in M, v \in \wedge_{k} T_{x} M$ and $\omega \in \wedge^{k} T_{x} M$ (see [F1, 1.7.8]).

Let $\mathscr{B}=(X, \pi, Z, Y, H)$ be a smooth fibre bundle. We assume that the total space $X$, the base space $Z$, and the fibre $Y$ are Riemannian manifolds of dimensions $n, n-m$ and $m$, respectively; that $Y$ is compact, connected, and orientable; that the metric on $Y$ is normalized so that $\mathcal{H}^{m}(Y)=1$; and that $H$ acts as a group of orientation preserving isometries of $Y$. Let $\omega_{0}$ be a unit orienting $m$-form for $Y$ and $\vec{Y}$ the $C^{\infty} m$-vectorfield dual to $\omega_{0}$. Then there exists a unique $m$-form $\Omega \in \mathcal{E}^{m}(X)$ such that

(1) $|\Omega|^{-1} \Omega(x)$ is dual to $\left|f_{\sharp} \vec{Y}\right|^{-1} f_{\sharp} \vec{Y}(x)$, and

(2) $|\Omega(x)|=\left|f_{\sharp} \vec{Y}(x)\right|^{-1}$

for every admissible map $f$ and $x \in \operatorname{im} f$. One also has

(3) $f^{\sharp} \Omega=\omega_{0}$.

Let $\Omega_{0}=|\Omega|^{-1} \Omega$ and $\vec{Y}_{0}=\gamma_{m}^{-1}\left(\Omega_{0}\right)$ be its dual. Note that (1) implies that $\vec{Y}_{0}(x)$ is simple and tangent to $\pi^{-1}\{\pi(x)\}$.

For $z \in Z$, denote

$$
Y_{z}=\mathcal{H}^{m}\left\llcorner\pi^{-1}\{z\} \wedge \vec{Y}_{0} \in I_{m}(X) .\right.
$$

We will also use $Y_{z}$ to denote $\pi^{-1}\{z\}=\operatorname{spt} Y_{z}$.

The set of all currents on a Riemannian manifold $U$ which are representable by integration will be denoted by $\mathbf{M}_{*}^{\text {loc }}(U)$; following [F2] we denote

$$
\mathbf{M}_{*}(U)=\mathbf{M}_{*}^{\text {loc }}(U) \cap \varepsilon_{*}(U) \text {. }
$$

Thus $T \in \mathbf{M}_{*}^{\text {loc }}(U)$ iff $T L \gamma \in \mathbf{M}_{*}(U)$ for all $\gamma \in \mathscr{D}^{0}(U)$.

If $V$ is an open subset of the Riemannian manifold $U$ and $T \in \mathscr{D}_{*}(U)$, then $T \mid V$ will denote the functional restriction of $T$ to $\mathscr{Q} *(V)$.

\section{Lifting currents representable by integration.}

3.1. Definition. The projection operator for the bundle $\mathscr{B}$ is the continuous linear map $P_{\mathscr{B}}: \mathscr{D}_{*}(X) \rightarrow \mathscr{D}_{*}(Z)$ of degree $-m$ defined by the formulae

$$
\begin{aligned}
& P_{\mathscr{B}}(T)=(-1)^{m(l-m)} \pi_{\sharp}\left(T\llcorner\Omega), \quad \text { if } T \in \mathscr{Q}_{l}(X) \text { with } m \leqslant l,\right. \\
& P_{\mathscr{B}}(T)=0, \quad \text { if } T \in \mathscr{D}_{l}(X) \text { with } 0 \leqslant l<m .
\end{aligned}
$$

Note that if $T \in \mathbf{M}_{k+m}^{\text {loc }}(X)$ and $\psi$ is a bounded Borel $l$-form on $Z$ with $l<k$, 
then $P_{\mathscr{B}}(T) \in \mathbf{M}_{k}^{\text {loc }}(Z)$ and

$$
P_{\mathscr{B}}\left(T\left\llcorner\pi^{\sharp} \psi\right)=\left(P_{\mathscr{B}} T\right)\llcorner\psi .\right.
$$

3.2. Let $L_{\mathscr{B}}^{\prime}: \mathbf{M}_{*}(Z) \rightarrow \mathbf{M}_{*}(X)$ be the unique linear operator such that:

(1) $L_{\mathscr{B}}^{\prime}(R)=R \times Y$ whenever $\mathscr{B}$ is a product bundle.

(2) $L^{\prime}$ is natural with respect to maps of bundles with fibre $Y$ and structure group $H$ (see [BJ1, 3.3]).

THEOREM. There exists a unique linear map

$$
L_{\mathscr{B}}: \mathbf{M}_{*}^{\mathrm{loc}}(Z) \rightarrow \mathbf{M}_{*}^{\mathrm{loc}}(X)
$$

such that

$$
L_{\mathscr{B}}(R L \gamma)=L_{\mathscr{B}}^{\prime}(R\llcorner\gamma)
$$

for every $R \in \mathbf{M}_{*}^{\mathrm{loc}}(Z)$ and $\gamma \in \mathscr{Q}^{0}(Z)$.

Proof. For $R \in \mathbf{M}_{k}^{\mathrm{loc}}(Z)$ and $\varphi \in \mathscr{Q}^{k+m}(X)$ define

$$
L_{\mathscr{B}} R(\varphi)=L_{\mathscr{B}}^{\prime}\left(R L \gamma_{\varphi}\right)(\varphi)
$$

where $\gamma_{\varphi} \in \mathscr{Q}^{0}(Z)$ is such that $\pi(\operatorname{spt} \varphi) \subset$ int $\gamma_{\varphi}^{-1}\{1\}$. One then uses linearity of $L_{\mathscr{B}}^{\prime}$ and [BJ1, 3.5(3), 3.6(2) and 3.5(2)] to show that $L_{\mathscr{B}} R(\varphi)$ is independent of the choice of $\gamma_{\varphi}$,

$$
L_{\mathscr{B}}(R L \gamma)=L_{\mathscr{B}}^{\prime}(R L \gamma)
$$

for $\gamma \in \mathscr{Q}^{0}(Z)$, and $L_{\mathscr{B}} R \in \mathbf{M}_{k+m}^{\text {loc }}(X)$. Uniqueness is obvious.

3.3. Corollary. (1) If $\mathscr{B}$ is a product bundle, then

$$
L_{\mathscr{B}} R=R \times Y \text { for } R \in \mathbf{M}_{*}^{\text {loc }}(Z) \text {. }
$$

(2) Let $\left\{R_{j}: j \in J\right\} \subset \mathbf{M}_{k}(Z)$ be such that $\left\{\right.$ spt $\left.R_{j}: j \in J\right\}$ is locally finite. Then

$$
R=\sum_{j \in J} R_{j} \in \mathbf{M}_{k}^{\mathrm{loc}}(Z) \text { and } L_{\mathscr{B}} R=\sum_{j \in J} L_{\mathscr{B}} R_{j} \text {. }
$$

(3) For any constant $c \in \mathbf{R}^{+}, L_{B}$ is continuous on

$$
\left\{R \in \mathbf{M}_{*}^{\text {loc }}(Z): \mathbf{M}(R)<c\right\} \text {. }
$$

(4) If $R \in \mathbf{M}_{k}^{\mathrm{loc}}(Z)$, then there exist a Baire $k$-vectorfield $\xi$ on $X$ and a Baire function $h \geqslant 0$ such that

$$
\left(L_{\mathscr{B}} R\right) \rightarrow(x)=\xi(x) \wedge \vec{Y}_{0}(x) \text { and } \pi_{\sharp}(h(x) \xi(x))=\vec{R}(\pi(x))
$$

for $\left\|L_{\mathscr{B}} R\right\|$ almost all $x \in X$.

(5) $P_{\mathscr{B}} \circ L_{\mathscr{B}}=\mathrm{id}_{\mathbf{M}_{*}^{\mathrm{loc}}(Z)}$.

(6) If $R \in \mathbf{M}_{k}^{\text {loc }}(Z)$ and $\psi$ is a bounded Borel l-form on $Z$ with $0 \leqslant l \leqslant k$, then

$$
L_{\mathscr{B}}(R L \psi)=\left(L_{\mathscr{B}} R\right)\left\llcorner\pi^{\sharp} \psi\right. \text {. }
$$

(7) For $R \in \mathbf{M}_{k}^{\text {loc }}(Z)$, spt $L_{\mathscr{B}} R=\pi^{-1}$ (spt $R$ ).

(8) The operator $L_{\mathscr{B}}$ can be uniquely extended to a linear operator on the subspace of $\mathscr{D}_{*}(Z)$ spanned by

$$
\left\{F \in \mathscr{D}_{*}(Z): F=\partial R \text { for some } R \in \mathbf{M}_{*}^{\mathrm{loc}}(Z)\right\} \cup \mathbf{M}_{*}^{\mathrm{loc}}(Z)
$$


such that $L_{\mathscr{B}} \circ \partial=\partial \circ L_{\mathscr{B}}$. Moreover, (7) holds for the extended operator.

(9) If $R \in \mathbf{F}_{k}^{\mathrm{loc}}(Z) \cap \mathbf{M}_{k}^{\mathrm{loc}}(Z)$ and $T \in \mathbf{F}_{k+m}^{\mathrm{loc}}(X) \cap \mathbf{M}_{k+m}^{\mathrm{loc}}(X)$, then

$$
L_{\mathscr{B}} R \in \mathbf{F}_{k+m}^{\mathrm{loc}}(X) \cap \mathbf{M}_{k+m}^{\mathrm{loc}}(X) \text { and } P_{\mathscr{B}} T \in \mathbf{F}_{k}^{\mathrm{loc}}(Z) \cap \mathbf{M}_{k}^{\mathrm{loc}}(Z) \text {. }
$$

Proof. We begin by establishing

$$
\left(L_{\mathscr{B}} R\right) L(\gamma \circ \pi)=L_{\mathscr{B}}(R L \gamma)
$$

for $\gamma \in \mathscr{Q}^{0}(Z)$. For $\varphi \in \mathscr{Q}^{k+m}(X)$ and $\gamma_{\varphi} \in \mathscr{Q}^{0}(Z)$ such that $\pi(\operatorname{spt} \varphi) \subset$ int $\gamma_{\varphi}^{-1}\{1\}$, one uses 3.2 and [BJ1, 3.6(2)] to see

$$
\begin{aligned}
\left(L_{\mathscr{B}} R\right)\llcorner(\gamma \circ \pi)(\varphi) & =L_{\mathscr{B}}^{\prime}\left(R L \gamma_{\varphi}\right)\left\llcorner(\gamma \circ \pi)(\varphi)=L_{\mathscr{B}}^{\prime}\left(R L \gamma_{\varphi} \gamma\right)(\varphi)\right. \\
& =L_{\mathscr{B}}(R L \gamma)(\varphi) .
\end{aligned}
$$

To prove (1), use (*), 3.2, [BJ1, 3.3(1)] and the equation

$$
R\left\llcorner\gamma \times Y=(R \times Y)\left\llcorner\pi^{\sharp} \gamma\right.\right.
$$

for $\gamma \in \mathscr{D}^{0}(Z)$.

For (2) observe that the function $\sum_{j \in J} R_{j}$ is a current, since $\left\{\varphi_{i}\right\} \subset \mathscr{D}^{k}(Z)$ converging to $\varphi_{0} \in \mathscr{Q}^{k}(Z)$ in $\mathscr{Q}^{k}(Z)$ implies $\cup_{j=0}^{\infty}$ spt $\varphi_{j}$ is contained in a compact set, and that $R \in \mathbf{M}_{k}^{\text {loc }}(Z)$, since $R_{j} \in \mathbf{M}_{k}(Z), j \in J$, and \{spt $R_{j}: j \in J$ \} is locally finite. If $\varphi \in \mathscr{Q}^{k+m}(X)$ and $\gamma_{\varphi} \in \mathscr{Q}^{0}(Z)$ with $\pi(\operatorname{spt} \varphi) \subset$ int $\gamma_{\varphi}^{-1}\{1\}$, then

$$
\begin{aligned}
L_{\mathscr{B}} R(\varphi) & =L_{\mathscr{B}}^{\prime}\left(R L \gamma_{\varphi}\right)(\varphi)=\sum_{j \in J} L_{\mathscr{B}}^{\prime}\left(R_{j} L \gamma_{\varphi}\right)(\varphi) \\
& =\sum_{j \in J} L_{\mathscr{B}} R_{j}(\varphi)
\end{aligned}
$$

by 3.2 and [BJ1, 3.3].

In order to verify (3) we choose a locally finite open cover $\left\{U_{j}\right\}$ of $Z$ such that $\operatorname{Clos}\left(U_{j}\right)$ is compact and let $\left\{\gamma_{j}\right\}$ be a partition of unity subordinate to $\left\{U_{j}\right\}$. Then if

$$
\left\{R_{\nu}\right\} \subset \mathscr{D}_{k}(Z) \cap\{R: \mathbf{M}(R) \leqslant c\}
$$

is a net converging to $R_{0}$, then

$$
L_{\mathscr{B}}\left(R _ { \nu } \llcorner \gamma _ { j } ) \rightarrow L _ { \mathscr { B } } \left(R_{0}\left\llcorner\gamma_{j}\right)\right.\right.
$$

by 3.2 and [BJ1, 3.5(1)]. Hence,

$$
\sum_{j} L_{\mathscr{B}}\left(R _ { \nu } \llcorner \gamma _ { j } ) \rightarrow \sum _ { j } L _ { \mathscr { B } } \left(R_{0}\left\llcorner\gamma_{j}\right)\right.\right.
$$

and the result now follows by (2).

(4) follows easily from (*), 3.2 and [BJ1, 3.5(5)].

Application of [BJ1, 3.6(1)], (*) and 3.1 yields (5).

For (6), let $R \in \mathbf{M}_{k}^{\text {loc }}(Z)$ and $\psi$ be a bounded Borel $l$-form with $0 \leqslant l \leqslant k$. Fix $\varphi \in \mathscr{D}^{k+m-l}(X)$ and let $\gamma_{\varphi} \in \mathscr{Q}^{0}(Z)$ be such that $\pi($ spt $\varphi) \subset$ int $\gamma_{\varphi}^{-1}\{1\}$. One uses [F1, 1.8.1] and [BJ1, 3.5(2)] to see that

$$
\mathbf{M}\left(L _ { \mathscr { B } } \left(R\left\llcorner\gamma_{\varphi}\right)\llcorner\varphi)<\infty\right.\right.
$$


Then by applying 3.2, [BJ1, 3.6(2)] and (*), one obtains

$$
\begin{aligned}
L_{\mathscr{B}}(R L \psi)(\varphi) & =L_{\mathscr{B}}^{\prime}\left(R L \gamma_{\varphi} \psi\right)(\varphi)=L_{\mathscr{B}}^{\prime}\left(R L \gamma_{\varphi}\right) L \pi^{\sharp} \psi(\varphi) \\
& =L_{\mathscr{B}} R \pi^{\sharp} \psi(\varphi) .
\end{aligned}
$$

Now (7) follows from (2), (6), [BJ1, 3.5(3)] and the observation that

$$
\text { spt } S=\bigcup_{j \in J} \text { spt } S\left\llcorner f_{j}\right.
$$

whenever $S \in \mathscr{D}_{k}(U)$ and $\left\{f_{j}: j \in J\right\}$ is a partition of unity subordinate to a locally finite open cover of a manifold $U$.

To verify (8) first observe that

$$
L_{\mathscr{B}} \partial R=\partial L_{\mathscr{B}} R
$$

for $R \in \mathbf{N}_{k}^{\text {loc }}(Z)$ by [BJ1, 3.5(4)], 3.2 and (6). Suppose $F=\partial R$ for some $R \in$ $\mathbf{M}_{k}^{\text {loc }}(Z)$. Define

$$
L_{\mathscr{B}}(F)=\partial L_{\mathscr{B}} R \text {. }
$$

To see that $L_{\mathscr{B}} F$ is well defined suppose $R_{1} \in \mathbf{M}_{k}^{\text {loc }}(Z)$ is such that $\partial R_{1}=F$. Let $\left\{U_{j}: j \in J\right\}$ be a locally finite open cover of $Z$ such that Clos $U_{j}$ is compact and there exist trivializations $\varphi_{j}: U_{j} \times Y \rightarrow \pi^{-1}\left(U_{j}\right)$, and let $\left\{\gamma_{j}\right\}$ be a partition of unity subordinate to $\left\{U_{j}: j \in J\right\}$. Note that 3.2, [BJ1, 3.3], $\partial Y=0$ and [F1, 4.1.8] imply

$$
\begin{aligned}
\partial L_{\mathscr{B}}\left(\left(R-R_{1}\right)\left\llcorner\gamma_{j}\right)\right. & =\partial \varphi_{j \sharp}\left(\left(R-R_{1}\right)\left\llcorner\gamma_{j} \times Y\right)\right. \\
& =-\varphi_{j \sharp}\left(\left(R-R_{1}\right)\left\llcorner d \gamma_{j} \times Y\right)\right. \\
& =-L_{\mathscr{B}}\left(\left(R-R_{1}\right)\left\llcorner d \gamma_{j}\right)\right.
\end{aligned}
$$

for each $j$. Apply (2) to obtain

$$
\begin{aligned}
\partial L_{\mathscr{B}}\left(R-R_{1}\right) & =\sum_{j \in J} \partial L_{\mathscr{B}}\left(\left(R-R_{1}\right)\left\llcorner\gamma_{j}\right)\right. \\
& =-\sum_{j \in J} L_{\mathscr{B}}\left(\left(R-R_{1}\right)\left\llcorner d \gamma_{j}\right)\right. \\
& =-L_{\mathscr{B}}\left(\left(R-R_{1}\right)\left\llcorner d\left(\sum_{j \in J} \gamma_{j}\right)\right)\right. \\
& =0 .
\end{aligned}
$$

Now $L_{\mathscr{B}}$ can be extended linearly.

The second statement in (8) follows from [F1, 4.18], local triviality and the fact that

$$
L_{\mathscr{B}} F(\varphi)=L_{\mathscr{B}}(F L \gamma)(\varphi)=\partial L_{\mathscr{B}}(R L \gamma)(\varphi)
$$

whenever $R \in \mathbf{M}_{l+1}^{\text {loc }}(Z), \quad F=\partial R, \quad \varphi \in \mathscr{D}^{l}(X), \quad \gamma \in \mathscr{Q}^{0}(Z)$ and spt $\varphi \subset$ $\operatorname{int}(\gamma \circ \pi)^{-1}\{1\}$.

To prove (9) let $R \in \mathbf{F}_{k}^{\text {loc }}(Z) \cap \mathbf{M}_{k}^{\text {loc }}(Z), \gamma \in \mathscr{D}^{0}(Z)$ and $C \subset Z$ be compact with spt $\gamma \subset$ int $C$. By [BJ1, 3.5(4)] and 3.2,

$$
L_{\mathscr{B}} R_{1} \in \mathbf{N}_{k+m}(X) \text { whenèter } R_{1} \in \mathbf{N}_{k}(Z) \text {, }
$$


so that one can use [F1, 4.1.17], 3.2 and [BJ1, 3.5(2) and (3)] to show

$$
L_{\mathscr{B}}(R L \gamma) \in \mathbf{F}_{k+m, \pi^{-1}(C)}(X) \cap \mathbf{M}_{k+m}(X),
$$

from which

$$
L_{\mathscr{B}} R \in \mathbf{F}_{k+m}^{\mathrm{loc}}(X) \cap \mathbf{M}_{k+m}^{\mathrm{loc}}(X)
$$

follows by (6). Similarly, [F1, 4.1.17] implies

$$
\left(T \llcorner \pi ^ { \# } \gamma ) \left\llcorner\Omega \in \mathbf{F}_{k, \pi^{-1}(C)}(X) \cap \mathbf{M}_{k}(X) .\right.\right.
$$

Since $\pi$ is proper, $[\mathbf{F 1}, 4.1 .14]$ implies

$$
P_{\mathscr{B}}\left(T\left\llcorner\pi^{\sharp} \gamma\right) \in \mathbf{F}_{k, C}(Z) \cap \mathbf{M}_{k}(X)\right.
$$

and (9) follows from 3.1.

3.4. TheOREM. Let $\iota(x): T_{\pi(x)} Z \rightarrow T_{x} X$ be the right inverse of $\pi_{\sharp} \mid T_{x} X$ such that $\iota(x)\left(T_{\pi(x)} Z\right)$ is the orthogonal complement of $T_{x} Y_{\pi(x)} \subset T_{x} X$. Let $R \in \mathbf{M}_{k}^{\text {loc }}(Z)$ and $h(x)=\left\|\wedge_{k} l(x) \vec{R}(\pi(x))\right\|$. Then

$$
\left\|L_{\mathscr{B}} R\right\|(f)=\int_{Z} \int_{Y_{z}} f h d \mathcal{H}^{m} d\|R\| z
$$

for each $\left\|L_{\mathscr{B}} R\right\|$ integrable Borel function $f$ on $X$.

Proof. Let $U$ be an open subset of $Z$ such that there exists a trivialization $\varphi: U \times Y \rightarrow \pi^{-1}(U)$, and whenever $u_{0} \in U, y_{0} \in Y$, let

$$
i_{y_{0}}: U \rightarrow U \times Y \text { and } j_{u_{0}}: Y \rightarrow U \times Y
$$

be the injections

$$
i_{y_{0}}(u)=\left(u, y_{0}\right) \text { and } j_{u_{0}}(y)=\left(u_{0}, y\right) .
$$

Suppose $R^{\prime} \in \mathbf{M}_{k}(U)$. By making the identification

$$
\bigwedge_{l} T_{(u, y)}(U \times Y) \cong \bigoplus_{r+s=l} \bigwedge_{r} T_{u}(U) \otimes \bigwedge_{s} T_{y}(Y)
$$

and applying [F1, 4.1.7 and 4.1.8] and [BJ1, 3.3], one obtains

$$
\begin{aligned}
\left\|L_{\mathscr{B}} R^{\prime}\right\| \wedge\left(L_{\mathscr{B}} R^{\prime}\right) & =L_{\mathscr{B}} R^{\prime}=\varphi_{\sharp}\left(R^{\prime} \times Y\right) \\
& =\varphi_{\sharp}\left(\left\|R^{\prime}\right\| \times\|Y\|\right) \wedge \varphi_{\sharp}\left(\vec{R}^{\prime} \otimes 1\right) \wedge \varphi_{\sharp}(1 \otimes \vec{Y}) .
\end{aligned}
$$

One uses (2) from $\$ 2$ to compute

$$
\left\|\left(\varphi \circ j_{u}\right)_{\sharp} \vec{Y}(y)\right\|=|\Omega(\varphi(u, y))|^{-1} .
$$

Also, if $x=\varphi(u, y)$, then

$$
\pi_{\sharp} \circ\left(\varphi \circ i_{y}\right)_{\sharp u}=\left(\pi \circ \varphi \circ i_{y}\right)_{\sharp u}=\mathrm{id}_{T_{u} U}
$$

implies $\operatorname{im}\left(\left(\varphi \circ i_{y}\right)_{\sharp}-\iota(x)\right) \subset \operatorname{ker} \pi_{\sharp}$ from which

$$
\left(\varphi \circ i_{y}\right)_{\sharp} \vec{R}^{\prime}(u) \wedge \vec{Y}_{0}(x)=\bigwedge_{k}(x)\left(\vec{R}^{\prime}(u)\right) \wedge \vec{Y}_{0}(x)
$$

follows. Hence,

$$
\left(L_{B} R^{\prime}\right) \rightarrow(x)=h(x)^{-1}|\Omega(x)|\left(\varphi \circ i_{y}\right)_{\sharp} \vec{R}^{\prime}(u) \wedge\left(\varphi \circ j_{u}\right)_{\sharp} \vec{Y}(y),
$$


so that

$$
\left\|L_{\mathscr{B}} R^{\prime}\right\|=\varphi_{\sharp}\left(\left\|R^{\prime}\right\| \times\|Y\|\right) \wedge h|\Omega|^{-1} .
$$

Now apply Fubini's theorem, the fact that $\|Y\|=\mathcal{H}^{m}$, a change of variables and 2(2) to obtain

$$
\begin{aligned}
\varphi_{\sharp}\left(\left\|R^{\prime}\right\| \times\|Y\|\right)(f) & =\int_{U} \int_{Y} f \circ \varphi(u, y) d\|Y\| y d\left\|R^{\prime}\right\| u \\
& =\int_{U} \int_{Y_{u}}|\Omega| f d \mathcal{F}^{m} d\left\|R^{\prime}\right\| u .
\end{aligned}
$$

The asserted formula for $R^{\prime}$ now follows, if we replace $f$ by $h|\Omega|^{-1} f$.

Suppose $R \in \mathbf{M}_{k}^{\text {loc }}(Z)$. Let $\left\{U_{j}\right\}$ be a locally finite open cover of $Z$ by coordinate neighborhoods in $\mathscr{B}$ such that $\operatorname{Clos} U_{j}$ is compact. Let $\left\{\gamma_{j}\right\} \subset \mathscr{D}^{0}(Z)$ be a partition of unity subordinate to $\left\{U_{j}\right\}$. Let $\left\{K_{i}\right\}_{i=1}^{\infty}$ be an increasing sequence of compact sets such that $\cup_{i=1}^{\infty} K_{i}=X$ and let $\chi_{i}$ denote the characteristic function of $K_{i}$. Then for each $\left\|L_{\mathscr{B}} R\right\|$ summable Borel function, one has

$$
\begin{aligned}
\left\|L_{\mathscr{B}} R\right\|\left(f \chi_{i}\right) & =\sum_{j}\left\|L_{\mathscr{B}} R\right\| L \pi^{\sharp} \gamma_{j}\left(f \chi_{i}\right) \\
& =\sum_{j}\left\|L_{\mathscr{B}}\left(R L \gamma_{j}\right)\right\|\left(f \chi_{i}\right) \\
& =\sum_{j} \int_{Z} \gamma_{j}(z) \int_{Y_{z}} f \chi_{i} h d \mathcal{F}^{m} d\|R\|(z) \\
& =\int_{Z} \int_{Y_{z}} f \chi_{i} h d \mathcal{H}^{m} d\|R\|(z)
\end{aligned}
$$

by local finiteness, 3.3(6) and the first paragraph. Using the monotone convergence theorem we conclude that

$$
\left\|L_{\mathscr{B}} R\right\|(f)=\int_{Z} \int_{Y_{z}} f h d \mathcal{F}^{m} d\|R\| z
$$

for nonnegative $f$, hence for arbitrary $f$ by the usual argument.

3.5. LeMMA. Let $U$ be a Riemannian manifold. If

$$
T \in \mathbf{F}_{k+m}^{\mathrm{loc}}(U \times Y) \cap \mathbf{M}_{k+m}^{\mathrm{loc}}(U \times Y)
$$

is such that

(1) for $\|T\|$ a.e. $(u, y) \in U \times Y$ there exists

$$
\xi(u, y) \in \bigwedge_{k} T_{(u, y)}(U \times Y)
$$

such that $\vec{T}=\xi \wedge \vec{Y}_{0}$, and

(2) $\partial T=0$, then there exists $R \in \mathbf{F}_{k}^{\mathrm{loc}}(U) \cap \mathbf{M}_{k}^{\mathrm{loc}}(U)$ such that

$$
T=R \times Y \text {. }
$$

Proof. Let $p: U \times Y \rightarrow U$ and $q: U \times Y \rightarrow Y$ be the projections. Let $\varphi \in$ $\mathscr{D}^{i}(U)$ and $\psi \in \mathscr{Q}^{j}(Y)$ where $i+j=k+m$. If $j<m$, then 


$$
T\left(p^{\sharp} \varphi \wedge q^{\sharp} \psi\right)=\int\left\langle\xi \wedge \vec{Y}_{0}, p^{\sharp} \varphi \wedge q^{\sharp} \psi\right\rangle d\|T\|=0 .
$$

Assume $j=m$ and fix $\varphi$. Since $\operatorname{spt}\left(T\left\llcorner p^{\#} \varphi\right)\right.$ is compact, one can use [F1, 4.1.17 and 4.1.14] to show that $q_{\sharp}\left(T\left\llcorner p^{\#} \varphi\right) \in \mathbf{F}_{m}(Y)\right.$. From the case $j<m$ and (2), one computes

$$
\partial q_{\sharp}\left(T L p^{\sharp} \varphi\right)=0 .
$$

By $[\mathbf{F 1}, 4.1 .31]$ there exists $c_{\varphi} \in \mathbf{R}$ such that

$$
q_{\sharp}\left(T\left\llcorner p^{\sharp} \varphi\right)=c_{\varphi} Y\right. \text {. }
$$

But then

$$
c_{\varphi}=c_{\varphi} Y\left(\omega_{0}\right)=(-1)^{m k} p_{\sharp}\left(T L q^{\sharp} \omega_{0}\right)(\varphi) .
$$

Letting $R=(-1)^{m k} p_{\sharp}\left(T\left\llcorner q^{\#} \omega_{0}\right)\right.$, one concludes that $R \in \mathbf{F}_{k}^{\text {loc }}(U) \cap \mathbf{M}_{k}^{\text {loc }}(U)$ and $R(\varphi)=c_{\varphi}$, hence

$$
T\left(p^{\sharp} \varphi \wedge q^{\sharp} \psi\right)= \begin{cases}R(\varphi) Y(\psi), & \text { if } m=j, \\ 0, & \text { if } m<j,\end{cases}
$$

from which the lemma follows by [F1, 4.1.8].

3.6. TheOREM. If $T \in \mathbf{F}_{k+m}^{\mathrm{loc}}(X) \cap \mathbf{M}_{k+m}^{\mathrm{loc}}(X)$ is such that

(1) for $\|T\|$ a.e. $x \in X$ there exists $\xi(x) \in \wedge_{k} T_{x}(X)$ such that

$$
\vec{T}=\xi \wedge \vec{Y}_{0}
$$

and

(2) $\mathcal{H}^{k+m}\left(\pi^{-1}(\pi(\operatorname{spt} \partial T))\right)=0$,

then $T=L_{\mathscr{B}} \circ P_{\mathscr{B}}(T)$.

Proof. Since $\pi$ is proper, $\pi(\operatorname{spt} \partial T)$ is closed. Let $\left\{U_{j}: j \in J\right\}$ be a locally finite open cover of

$$
Z \sim \pi(\operatorname{spt} \partial T)
$$

such that each $U_{j}$ is a coordinate neighborhood for $\mathscr{B}$ with trivialization $\varphi_{j}: U_{j} \times Y \rightarrow \pi^{-1}\left(U_{j}\right)$ and Clos $U_{j}$ is compact. Let $\left\{\gamma_{j}: j \in J\right\}$ be a partition of unity subordinate to $\left\{U_{j}: j \in J\right\}$ and denote $V_{j}=\pi^{-1}\left(U_{j}\right)$ and

$$
T_{j}=T \mid V_{j} \in \mathbf{F}_{k+m}^{\mathrm{loc}}\left(V_{j}\right) \cap \mathbf{M}_{k+m}^{\mathrm{loc}}\left(V_{j}\right) .
$$

According to [F1, 4.1.14],

$$
\varphi_{j \sharp}^{-1} T_{j} \in \mathbf{F}_{k+m}^{\mathrm{loc}}\left(U_{j} \times Y\right) \cap \mathbf{M}_{k+m}^{\mathrm{loc}}\left(U_{j} \times Y\right),
$$

so that an application of 3.5 gives $R_{j} \in \mathbf{F}_{k}^{\text {loc }}\left(U_{j}\right) \cap \mathbf{M}_{k}^{\text {loc }}\left(U_{j}\right)$ such that

$$
T_{j}=\varphi_{j \sharp}\left(R_{j} \times Y\right) \text {. }
$$

Thus by [BJ1, 3.3] and 3.2,

$$
\begin{aligned}
T\left\llcorner\pi^{\sharp} \gamma_{j}\right. & =T_{j} \mathrm{~L} \pi^{\sharp} \gamma_{j}=\varphi_{j \sharp}\left(R_{j} \times Y\right)\left\llcorner\pi^{\sharp} \gamma_{j}\right. \\
& =\varphi_{j \sharp}\left(R_{j}\left\llcorner\gamma_{j} \times Y\right)=L_{\mathscr{B}}\left(R_{j} \mathrm{~L} \gamma_{j}\right) .\right.
\end{aligned}
$$


Hence

$$
T\left\llcorner\pi^{\sharp} \gamma_{j}=L_{\mathscr{B}} \circ P_{\mathscr{B}}\left(T\left\llcorner\pi^{\sharp} \gamma_{j}\right)=\left(L_{\mathscr{B}} \circ P_{\mathscr{B}} T\right)\left\llcorner\pi^{\sharp} \gamma_{j}\right.\right.\right.
$$

now follows from 3.3(5), 3.1 and 3.3(6), and we conclude that

$$
\operatorname{spt}\left(T-L_{\mathscr{B}} \circ P_{\mathscr{B}} T\right) \subset \pi^{-1}(\pi \text { spt } \partial T) .
$$

Finally, $L_{\mathscr{B}} \circ P_{\mathscr{B}} T \in \mathbf{F}_{k+m}^{\text {loc }}(X)$ and application of [F1, 2.10.6 and 4.1.20] yields

$$
T=L_{\mathscr{B}} \circ P_{\mathscr{B}} T \text {. }
$$

\section{Projecting then lifting in the bundle induced by a group action.}

4.1. Let $G$ be a compact, connected Lie group of isometries of $X$. Let $\mu$ be the biinvariant Haar measure such that $\mu(G)=1$. If we assume that there is only one orbit type, then $\mathscr{B}=\left(X, \pi, X / G, G / G_{0}, \Re\left(G_{0}\right) / G_{0}\right)$ is a smooth bundle where $G_{0}=\left\{g \in G: g x_{0}=x_{0}\right\}$ for some fixed $x_{0}$ and $\mathscr{\Re}\left(G_{0}\right)$ is the normalizer of $G_{0}$ in $G$ [BG, II.5.8]. Assume that $X$ and $X / G$ are orientable. Then $\mathscr{B}$ and $G / G_{0}$ are orientable, and $\Re\left(G_{0}\right) / G_{0}$ can be replaced by the subgroup $H$ consisting of those elements which preserve an orientation of $G / G_{0}$. Let $Z=X / G, Y=G / G_{0}$ and $\omega_{0}, \Omega, \vec{Y}_{0}$ be as before.

For such a bundle every local trivialization $\varphi: U \times Y \rightarrow \pi^{-1}(U)$ is equivariant. To see this choose an equivariant trivialization $\varphi_{U}$ as in [BG, II.5.8] and note that for fixed $u \in U$ there exists $h \in \mathfrak{N}\left(G_{0}\right) / G_{0}$ such that

$$
p \circ \varphi_{U}^{-1} \circ \varphi \circ i_{u}: Y \rightarrow Y
$$

is given by the action of $h$, where $i_{u}: Y \rightarrow U \times Y$ is the injection over $u$ and $p: U \times Y \rightarrow Y$ is the projection onto the second factor. A straightforward computation yields the equivariance of $\varphi_{U}^{-1} \circ \varphi$, from which the equivariances of $\varphi$ follows. Note that this also implies that admissible maps are equivariant (see [B, 3.2]).

The manifold $Z$ has a natural Riemannian metric $b_{\pi}$ induced by $\pi$ as follows: For $z \in Z$ and $w_{1}, w_{2} \in T_{z} Z$ choose $x \in \pi^{-1}\{u\}$ and let $v_{1}, v_{2} \in T_{x} X$ be the unique vectors in the orthogonal complement of ker $\pi_{\sharp} \mid T_{x} X$ such that

$$
\pi_{\sharp}\left(v_{i}\right)=w_{i}, \quad i=1,2 .
$$

Define

$$
b_{\pi}\left(w_{1}, w_{2}\right)=b\left(v_{1}, v_{2}\right)
$$

where $b: T X \times T X \rightarrow \mathbf{R}$ is the metric on $X$. Since $G$ is a group of isometries of $X$, $b_{\pi}\left(w_{1}, w_{2}\right)$ does not depend on the choice of $x$ and the smoothness of $b_{\pi}$ follows from the existence of local cross sections.

Let $\vec{X}$ be a unit $C^{\infty} n$-vectorfield on $X$ and use $\vec{X}$ to define a Hodge duality operator *. Let $\beta \in \mathcal{E}^{n-m}(Z)$ be nonvanishing. Let $x \in X, z=\pi(x)$ and choose an admissible map $f: Y \rightarrow X$ such that $\pi \circ f \equiv z$. Denoting

$$
k(x)=\left\langle f_{\sharp} \vec{Y}(x), * \pi^{\sharp} \beta(x)\right\rangle,
$$

one obtains from 2(2) and 2(3),

$$
\Omega(x)=k(x)^{-1} * \pi^{\sharp} \beta(x) .
$$


Since admissible maps are equivariant and $G$ is connected, it follows from the above expression that $\Omega$ is $G$-invariant.

We now define the volume functions

$$
V: X \rightarrow \mathbf{R} \text { and } V_{\pi}: Z \rightarrow \mathbf{R}
$$

by the formula

$$
V_{\pi}(z)=V(x)=|\Omega(x)|^{-1}
$$

where $\pi(x)=z$. That $V_{\pi}$ is well defined follows from the $G$-invariance of $\Omega$. Note that 2(1) and (3) imply

$$
\begin{aligned}
\mathcal{F}^{m}\left(Y_{z}\right) & =Y_{z}\left(\Omega_{0}\right)=Y_{z}(V \wedge \Omega) \\
& =Y\left(V_{\pi}(z) \wedge \omega_{0}\right)=V_{\pi}(z) .
\end{aligned}
$$

4.2. TheOREM. If $T \in \mathbf{M}_{k+m}^{\text {loc }}(X)$, then

$$
L_{\mathscr{B}} \circ P_{\mathscr{B}} T=T
$$

if and only if

(1) $g_{\sharp} T=T$ for all $g \in G$, and

(2) for $\|T\|$ almost all $x \in X$ there exists $v \in \wedge_{k} T_{x} X$ such that $\vec{T}(x)=$ $v \wedge \vec{Y}_{0}(x)$.

Proof. If $L_{\mathscr{B}} \circ P_{\mathscr{B}} T=T$, then (2) is just 3.3(4). To prove (1) use a partition of unity, 3.3(2), 3.2 and [BJ1, 3.3] to infer that it suffices to show

$$
g_{\sharp} \varphi_{\sharp}(R \times Y)=\varphi_{\sharp}(R \times Y)
$$

for $g \in G$ where spt $R$ is contained in a coordinate neighborhood $U$ of $\mathscr{B}$ and $\varphi: U \times Y \rightarrow X$ is a trivialization. But the equation follows from the equivariance of $\varphi$ and the fact that $g_{\sharp} Y=Y$.

Now suppose $T$ satisfies (1) and (2). Let $\left\{U_{j}\right\}$ be a locally finite open cover of $Z$ by coordinate neighborhoods of $\mathscr{B}$ having compact closure, $\varphi_{j}: U_{j} \times Y \rightarrow \pi^{-1}\left(U_{j}\right)$ be trivializations, and $\left\{\gamma_{j}\right\} \subset \mathscr{Q}^{0}(Z)$ be a partition of unity subordinate to $\left\{U_{j}\right\}$. Define

$$
T_{j}=\varphi_{j \sharp}^{-1}\left(T\left\llcorner\pi^{\sharp} \gamma_{j}\right) .\right.
$$

Fix $j$ and $\psi \in \mathscr{Q}^{k}\left(U_{j}\right)$ and consider

$$
q_{\sharp}\left(T _ { j } \llcorner p ^ { \sharp } \psi ) \left\llcorner\omega_{0}\right.\right.
$$

where $p: U_{j} \times Y \rightarrow U_{j}$ and $q: U_{j} \times Y \rightarrow Y$ are the projections. Since $\varphi_{j}^{-1}$ is equivariant, $T$ is invariant, and $\omega_{0}$ is invariant, we have

$$
\left(\text { id }_{U_{j}} \times g\right)_{\sharp} T_{j}=T_{j}
$$

and

$$
\begin{aligned}
g_{\sharp}\left(q _ { \sharp } \left(T_{j}\left\llcorner p^{\sharp} \psi\right)\left\llcorner\omega_{0}\right)\right.\right. & =q_{\sharp}\left(\operatorname{id}_{U_{j}} \times g\right)_{\sharp}\left(T _ { j } \llcorner p ^ { \sharp } \psi ) \left\llcorner\omega_{0}\right.\right. \\
& =q_{\sharp}\left(T _ { j } \llcorner p ^ { \sharp } \psi ) \left\llcorner\omega_{0} .\right.\right.
\end{aligned}
$$

Thus $q_{\sharp}\left(T_{j}\left\llcorner p^{\sharp} \psi\right)\left\llcorner\omega_{0}\right.\right.$ is a $G$-invariant Radon measure on $Y$ and there exists $c_{\psi} \in \mathbf{R}$ 
such that

We have

$$
q_{\sharp}\left(T _ { j } \llcorner p ^ { \sharp } \psi ) \left\llcorner\omega_{0}=c_{\psi} \mathcal{H}^{m}=c_{\psi} Y\left\llcorner\omega_{0} .\right.\right.\right.
$$

$$
\begin{aligned}
c_{\psi} & =c_{\psi} Y\left(\omega_{0}\right)=T_{j}\left(p^{\sharp} \psi \wedge q^{\sharp} \omega_{0}\right) \\
& =T\left(\pi^{\sharp}\left(\gamma_{j} \wedge \psi\right) \wedge\left(q \circ \varphi_{j}^{-1}\right)^{\sharp} \omega_{0}\right) .
\end{aligned}
$$

By 2(2) and (1) we have

$$
\left\langle\vec{Y}_{0}(x),\left(q \circ \varphi_{j}^{-1}\right)^{\#} \omega_{0}(x)\right\rangle=\left|\left(\varphi_{j} \circ i_{z}\right)_{\sharp} \vec{Y}(x)\right|^{-1}=\left\langle\vec{Y}_{0}(x), \Omega(x)\right\rangle,
$$

where $\pi(x)=z$ and $i_{z}: Y \rightarrow U_{j} \times Y$ is the injection $i_{z}(y)=(z, y)$. Now use the simplicity of $\vec{Y}_{0}$ to obtain

$$
\left\langle v \wedge \vec{Y}_{0}(x), \pi^{\sharp}\left(\gamma_{j} \wedge \psi\right) \wedge\left(q \circ \varphi_{j}^{-1}\right)^{\sharp} \omega_{0}(x)\right\rangle=\left\langle v \wedge \vec{Y}_{0}(x), \pi^{\sharp}\left(\gamma_{j} \wedge \psi\right) \wedge \Omega(x)\right\rangle,
$$

so that

$$
c_{\psi}=P_{\mathscr{B}} T L \gamma_{j}(\psi) \text {. }
$$

For $\omega \in \mathscr{D}^{m}(Y)$ there exists $h \in \mathscr{Q}^{0}(Y)$ such that $\omega=h \omega_{0}$ and hence

$$
\begin{aligned}
P_{\mathscr{B}} T L \gamma_{j} \times Y\left(p^{\sharp} \psi \wedge q^{\sharp} \omega\right) & =P_{\mathscr{B}} T\left\llcorner\gamma_{j}(\psi) Y\left(h \wedge \omega_{0}\right)=c_{\psi} Y L \omega_{0}(h)\right. \\
& =q_{\sharp}\left(T _ { j } \llcorner p ^ { \sharp } \psi ) \left\llcorner\omega_{0}(h)=T_{j}\left(p^{\sharp} \psi \wedge q^{\sharp} \omega\right) .\right.\right.
\end{aligned}
$$

Using (2) to infer that for $\psi \in \mathscr{Q}^{k+l}\left(U_{j}\right)$ and $\omega \in \mathscr{D}^{m-l}(Y)$ with $l>0$, the equation

$$
T_{j}\left(p^{\sharp} \psi \wedge q^{\sharp} \omega\right)=T\left(\pi^{\sharp}\left(\gamma_{j} \wedge \psi\right) \wedge\left(q^{\circ} \varphi_{j}^{-1}\right)^{\sharp} \omega\right)=0
$$

holds, we have

$$
T_{j}=\left(P_{\mathscr{B}} T\left\llcorner\gamma_{j}\right) \times Y .\right.
$$

Thus by [BJ1, 3.3], 3.2 and 3.3(6), we conclude

$$
\begin{aligned}
T & =\sum_{j} T\left\llcorner\pi^{\sharp} \gamma_{j}=\sum_{j} \varphi_{j \sharp} T_{j}\right. \\
& =\sum_{j} \varphi_{j \sharp}\left(\left(P_{\mathscr{B}} T\left\llcorner\gamma_{j}\right) \times Y\right)=\sum_{j} L_{\mathscr{B}}\left(P_{\mathscr{B}} T\left\llcorner\gamma_{j}\right)\right.\right. \\
& =\sum_{j}\left(L_{\mathscr{B}} \circ P_{\mathscr{B}} T\right)\left\llcorner\pi^{\sharp} \gamma_{j}=L_{\mathscr{B}} \circ P_{\mathscr{B}} T .\right.
\end{aligned}
$$

4.3. REMARK. It is easy to see that for locally rectifiable currents $T$, condition (1) of 4.2 implies condition (2). However, there are simple examples of normal currents in $X=\mathbf{R} \times S^{1}$ which satisfy (1) but not (2).

4.4. TheOREM. For each $T \in \mathbf{M}_{l}^{\mathrm{loc}}(X)$ and $\varphi \in \mathscr{D}^{l}(X)$, let

$$
A(T)(\varphi)=\int_{G} g_{\sharp} T(\varphi) d \mu g .
$$


Then:

(1) If $\mathfrak{T}$ is the linear subspace of $\mathscr{D}_{*}(X)$ spanned by

$$
\left\{Q \in \mathscr{D}_{*}(X): Q=\partial T \text { for some } T \in \mathbf{M}_{*}^{\mathrm{loc}}(X)\right\} \cup \mathbf{M}_{*}^{\mathrm{loc}}(X),
$$

then $A$ can be extended to a chain map $A: \mathfrak{T} \rightarrow \mathscr{D}_{*}(X)$.

(2) If $T \in \mathbf{M}_{l}^{\text {loc }}(X)$ and $\gamma \in \mathscr{Q}^{0}(Z)$, then

$$
\mathbf{M}\left(A\left(T\left\llcorner\pi^{\sharp} \gamma\right)\right) \leqslant \mathbf{M}\left(T\left\llcorner\pi^{\sharp} \gamma\right) .\right.\right.
$$

(3) If $T \in \mathbf{F}_{l}^{\mathrm{loc}}(X) \cap \mathbf{M}_{l}^{\mathrm{loc}}(X)$, then $A(T) \in \mathbf{F}_{l}^{\mathrm{loc}}(X) \cap \mathbf{M}_{l}^{\mathrm{loc}}(X)$.

Proof. If $T^{\prime} \in \mathbf{M}_{l}(X)$ and $\varphi \in \mathscr{D}^{l}(X)$, then

$$
\left|T^{\prime}\left(g^{\sharp} \varphi\right)\right| \leqslant \mathbf{M}\left(T^{\prime}\right) \mathbf{M}\left(g^{\sharp} \varphi\right)=\mathbf{M}\left(T^{\prime}\right) \mathbf{M}(\varphi),
$$

hence

$$
\left|A\left(T^{\prime}\right)(\varphi)\right| \leqslant \int_{G}\left|g_{\sharp} T^{\prime}(\varphi)\right| d \mu \leqslant \mathbf{M}\left(T^{\prime}\right) \mathbf{M}(\varphi) .
$$

By replacing $T^{\prime}$ by $T L \pi^{\sharp} \gamma$ for $T \in \mathbf{M}_{l}^{\text {loc }}(X)$ and $\gamma \in \mathscr{D}^{0}(Z)$, one obtains

$$
A: \mathbf{M}_{*}^{\mathrm{loc}}(X) \rightarrow \mathbf{M}_{*}^{\mathrm{loc}}(X)
$$

and (2). It is easy to see that $A$ can be extended to $\mathscr{N}$ by use of the equation

$$
A(\partial T)=\partial A(T) \text {. }
$$

Statement (3) follows from (1), (2), [F1, 4.1.17] and the fact that

$$
A(T)\llcorner\beta=A(T\llcorner\beta)
$$

for $G$-invariant $\beta \in \mathscr{D}^{0}(X)$.

4.5. Lemma. Let $W$ be a finite dimensional vector space and $v \in \wedge_{k+m} W$ and $\omega \in \wedge^{m} W$ be nonzero and simple. Then:

(1) $v \mathrm{~L} \omega$ is simple.

(2) If $L$ is the linear subspace associated with $v, \omega=\omega^{1} \wedge \cdots \wedge \omega^{m}$ where $\omega^{j} \in \wedge^{1} W, j=1, \ldots, m$, and $v\llcorner\omega \neq 0$, then

$$
L^{\prime}=L \cap \bigcap_{j=1}^{m} \operatorname{ker} \omega^{j}
$$

has dimension $k$.

(3) If $v L \omega \neq 0$, and $v^{\prime} \in \bigwedge_{k} L^{\prime}$ and $v^{\prime \prime} \in \bigwedge_{m}$ L are such that $v^{\prime} \wedge v^{\prime \prime}=v$, then

$$
v L \omega=(-1)^{m k}\left\langle v^{\prime \prime}, \omega\right\rangle v^{\prime} \text {. }
$$

Proof. Fix an inner product $b$ on $W$. Let $w_{j} \in W$ be dual to $\omega^{j}, j=1, \ldots, m$, so that $w=w_{1} \wedge \cdots \wedge w_{m}$ is dual to $\omega$. Let $W_{0}$ be the subspace of $W$ associated with $w$. Denote by $p_{0}$ the orthogonal projection $p_{0}: W \rightarrow W_{0}$ and $p_{1}=p_{0} \mid L$. Since $p_{0}$ is orthogonal, $\left\{w_{j}: j=1, \ldots, m\right\}$ is a basis for $W_{0}$, and $\omega^{j}$ is dual to $w_{j}$, we have

$$
\begin{aligned}
v_{0} \in \operatorname{ker} p_{0} & \Leftrightarrow b\left(v_{0}, w_{j}\right)=0, \quad j=1, \ldots, m, \\
& \Leftrightarrow v_{0} \in \bigcap_{j=1}^{m} \operatorname{ker} \omega^{j} .
\end{aligned}
$$


Hence

$$
\operatorname{ker} p_{1}=L \cap \operatorname{ker} p_{0}=L^{\prime}
$$

Let $l=\operatorname{dim} L^{\prime}$. Clearly $l \geqslant k$.

Choose an orthonormal basis $\left\{\varepsilon_{1}, \ldots, \varepsilon_{n}\right\}$ of $W$ such that $\left\{\varepsilon_{1}, \ldots, \varepsilon_{l}\right\}$ spans $\operatorname{ker} p_{1}$ and $\left\{\varepsilon_{1}, \ldots, \varepsilon_{k+m}\right\}$ spans $L$. For some $c \in \mathbf{R}$ we have $v=$ $c \varepsilon_{1} \wedge \cdots \wedge \varepsilon_{m+k}$. For each $\lambda \in \Lambda(k+m, m)$ we associate $\lambda^{\prime} \in \Lambda(k+m, k)$ by requiring $\operatorname{im} \lambda \cup \operatorname{im} \lambda^{\prime}=\{1, \ldots, m+k\}$. If $\iota=\operatorname{id}_{\{1, \ldots, m+k\}}$ and $\nu \in \Lambda(n, k)$, then

$$
\begin{aligned}
\left\langle v L \omega, \varepsilon^{\nu}\right\rangle & =\left\langle c \varepsilon_{1} \wedge \cdots \wedge \varepsilon_{k+m}, \omega \wedge \varepsilon^{\nu}\right\rangle \\
& =\sum_{\lambda \in \Lambda(k+m, m)} \delta_{\lambda, \lambda^{\prime}}^{\iota} c\left\langle\varepsilon_{\lambda}, \omega\right\rangle\left\langle\varepsilon_{\lambda^{\prime}}, \varepsilon^{\nu}\right\rangle \\
& =\sum_{\lambda \in \Lambda(k+m, m)} \delta_{\lambda, \lambda^{\prime}}^{\iota} \delta_{\lambda^{\prime}}^{\nu} c\left\langle\varepsilon_{\lambda}, \omega\right\rangle .
\end{aligned}
$$

But if $\lambda \in \Lambda(k+m, m)$ is such that $\lambda(1) \leqslant l$ then $\varepsilon_{\lambda(1)} \in \operatorname{ker} p_{1} \subset \bigcap_{j=1}^{m} \operatorname{ker} \omega^{j}$ so that

$$
\left\langle\varepsilon_{\lambda}, \omega\right\rangle=0
$$

Hence $v L \omega=0$ unless $l \leqslant k$, in which case we have

$$
v L \omega=(-1)^{m k} c\left\langle\varepsilon_{k+1} \wedge \cdots \wedge \varepsilon_{k+m}, \omega\right\rangle \varepsilon_{1} \wedge \cdots \wedge \varepsilon_{k}
$$

Statement (3) now follows easily from the observation that, for $v^{\prime} \in \wedge_{k} L^{\prime}$, there exists $c^{\prime} \in \mathbf{R}$ such that

$$
v^{\prime}=c^{\prime} \varepsilon_{1} \wedge \cdots \wedge \varepsilon_{k} .
$$

4.6. Lemma. Let $W$ be an $n$-dimensional vector space with basis $\left\{w_{1}, \ldots, w_{n}\right\}$. If $\left\{\omega^{1}, \ldots, \omega^{n}\right\} \subset \wedge^{1} W$ is the dual basis, $w=w_{1} \wedge \cdots \wedge w_{m}$ and $\omega=\omega^{1}$ $\wedge \cdots \wedge \omega^{m}$, then

$$
\left(( \beta \llcorner \omega ) \wedge w ) \left\llcorner\omega=(-1)^{m k} \beta\llcorner\omega\right.\right.
$$

and

$$
\left((\alpha \wedge w)\llcorner\omega) \wedge w=(-1)^{m k} \alpha \wedge w\right.
$$

for all $\alpha \in \wedge_{k} W$ and $\beta \in \wedge_{k+m} W$. Moreover, if $W$ has an inner product and the spaces spanned by $\left\{w_{1}, \ldots, w_{m}\right\}$ and $\left\{w_{m+1}, \ldots, w_{n}\right\}$ are orthogonal, then

$$
\mid(\beta\llcorner\omega) \wedge w|\leqslant| \beta \mid
$$

with equality if and only if

$$
\beta=(-1)^{m k}(\beta L \omega) \wedge w .
$$

Proof. Let $\lambda_{0} \in \Lambda(n, m)$ be defined by

$$
\lambda_{0}(i)=i
$$

and for $\nu \in \Lambda(n, k)$ denote by $\nu^{\prime}$ the function from $\{m+1, \ldots, m+k\}$ to $\{1, \ldots, n\}$ defined by the formula

$$
\nu^{\prime}(i)=\nu(i-m)
$$


If $\lambda \in \Lambda(n, m+k)$ and $\nu \in \Lambda(n, k)$, then

$$
\left\langle w_{\lambda} L \omega, \omega^{\nu}\right\rangle=\delta_{\lambda}^{\lambda_{0} \cup \nu^{\prime}}
$$

Thus if $\lambda_{0} \nsubseteq \lambda$, then

$$
w_{\lambda} L \omega=0=(-1)^{m k}\left(\left(w_{\lambda} L \omega\right) \wedge w\right)\llcorner\omega .
$$

On the other hand if $\lambda=\lambda_{0} \cup \nu^{\prime}$, then

Thus,

$$
w_{\lambda} L \omega=w_{\nu}
$$

$$
\left(\left(w_{\lambda} L \omega\right) \wedge w\right)\left\llcorner\omega=\left(w_{\nu} \wedge w\right)\left\llcorner\omega=(-1)^{m k} w_{\lambda} L \omega .\right.\right.
$$

The second assertion follows similarly.

Finally, let $B=\left\{\lambda \in \Lambda(n, m+k): \lambda_{0} \nsubseteq \lambda\right\}$. Then for $\beta \in \wedge_{k+m} W$,

$$
\beta=\sum_{\lambda \in B} \beta^{\lambda} w_{\lambda}+\sum_{\nu \in \Lambda(n-m, k)} \beta^{\lambda_{0} \cup \nu^{\prime}} w \wedge w_{\nu+m}
$$

Hence,

$$
(\beta L \omega) \wedge w=(-1)^{m k} \sum_{\nu \in \Lambda(n-m, k)} \beta^{\lambda_{0} \cup v^{\prime}} w \wedge w_{\nu+m},
$$

and since the space spanned by $\left\{w_{1}, \ldots, w_{m}\right\}$ is orthogonal to the space spanned by $\left\{w_{m+1}, \ldots, w_{n}\right\}$, we conclude

$$
|\beta|=|(\beta L \omega) \wedge w| \text { if and only if } \beta^{\lambda}=0 \text { for } \lambda \in B \text {. }
$$

4.7. LEMMA. Let $W$ be an n-dimensional vector space with an inner product. Suppose $w \in \wedge_{m} W$ and $\omega \in \wedge^{m} W$ are simple, of norm one, and dual. If $\xi_{1} \in$ $\bigwedge_{k+m} W$ and

$$
\xi_{2}=(-1)^{m k}\left(\xi_{1} L \omega\right) \wedge w
$$

then

$$
\left\|\xi_{1}\right\|=\left\|\xi_{2}\right\| \text { if and only if } \xi_{2}=\xi_{1} \text {. }
$$

Proof. Assume $\left\|\xi_{2}\right\|=\left\|\xi_{1}\right\| \neq 0$. Use [F1, 1.8.1] to find simple $(k+m)$-vectors $v_{i} \neq 0, i=1, \ldots, N$, such that

$$
\xi_{1}=\sum_{i=1}^{N} v_{i} \text { and }\left\|\xi_{1}\right\|=\sum_{i=1}^{N}\left|v_{i}\right|
$$

Denote

$$
c_{i}=\left|v_{i}\right| /\left\|\xi_{1}\right\| \quad \text { and } \quad u_{i}=c_{i}^{-1} v_{i}, \quad i=1, \ldots, N
$$

From

$$
\xi_{2}=(-1)^{m k} \sum_{i=1}^{N} c_{i}\left(u_{i} \mathrm{~L} \omega\right) \wedge w
$$

and 4.5(1) we obtain

$$
\left\|\xi_{2}\right\| \leqslant \sum_{i=1}^{N} c_{i}\left|\left(u_{i} L \omega\right) \wedge w\right|
$$


Observe that $\left\|\xi_{1}\right\|=\left|u_{i}\right|$ for each $i$ and apply 4.6 to conclude

$$
\left\|\xi_{2}\right\| \leqslant \sum_{i=1}^{N} c_{i}\left|u_{i}\right|=\sum_{i=1}^{N} c_{i}\left\|\xi_{1}\right\|=\left\|\xi_{1}\right\|=\left\|\xi_{2}\right\| .
$$

Thus $\left|u_{i}\right|=\left|\left(u_{i} L \omega\right) \wedge w\right|$ and 4.6 implies

$$
u_{i}=(-1)^{m k}\left(u_{i} \mathrm{~L} \omega\right) \wedge w
$$

from which the lemma follows.

4.8. TheOREM. Let $T_{0} \in \mathbf{M}_{k+m}^{\text {loc }}(X)$. For $\left\|T_{0}\right\|$ almost every $x \in X$ denote

$$
\xi_{1}(x)=(-1)^{m k}\left(\vec{T}_{0}(x)\left\llcorner\Omega_{0}(x)\right) \wedge \vec{Y}_{0}(x)\right.
$$

and let

$$
T_{1}=\left\|T_{0}\right\| \wedge \xi_{1} \text { and } T_{2}=A\left(T_{1}\right)
$$

Then:

(1) If $\gamma \in \mathscr{D}^{0}(Z)$, then

$$
\mathbf{M}\left(T_{2}\left\llcorner\pi^{\sharp} \gamma\right) \leqslant \mathbf{M}\left(T_{1}\left\llcorner\pi^{\sharp} \gamma\right) \leqslant \mathbf{M}\left(T_{0}\left\llcorner\pi^{\sharp} \gamma\right) .\right.\right.\right.
$$

(2) If $\mathbf{M}\left(T_{0}\right)<\infty$, then

$$
\mathbf{M}\left(T_{1}\right)=\mathbf{M}\left(T_{0}\right) \text { if and only if } T_{1}=T_{0} .
$$

(3) $L_{\mathscr{B}} \circ P_{\mathscr{B}}\left(T_{i}\right)=T_{2}, i=0,1,2$.

Proof. Statements (1) and (2) follow from 4.4(2), the fact that

$$
\mathbf{M}\left(\left\|T_{0}\right\| \wedge\left(\pi^{\sharp} \gamma\right) \xi_{1}\right)=\int \pi^{\sharp} \gamma\left\|\xi_{1}\right\| d\left\|T_{0}\right\|,
$$

the simplicity of $\Omega_{0}, 2(1)$ and 4.7 .

Using the $G$-invariance of $\Omega, \Omega_{0}$ and $\vec{Y}_{0}$, the duality of $\Omega_{0}$ and $\vec{Y}_{0}$, and 4.6 one obtains the equalities

$$
P_{\mathscr{B}} T_{0}=P_{\mathscr{B}} T_{1}=P_{\mathscr{B}} T_{2}
$$

and

$$
\begin{aligned}
T_{2}(\varphi) & =\int_{G} \int\left\langle\vec{T}_{1}, g^{\sharp} \varphi\right\rangle d\left\|T_{0}\right\| d \mu g \\
& \left.=\int_{G} \int\left\langle\vec{T}_{1}, g^{\sharp}\left(\vec{Y}_{0}\right\lrcorner \varphi\right) \wedge \Omega_{0}\right\rangle d\left\|T_{0}\right\| d \mu g \\
& \left.=T_{2}\left(\left(\vec{Y}_{0}\right\lrcorner \varphi\right) \wedge \Omega_{0}\right)
\end{aligned}
$$

for all $\varphi \in \mathscr{Q}^{k+m}(X)$. The last equality implies that $T_{2}$ satisfies $4.2(2)$ and since $T_{2}$ is obviously $G$-invariant, an application of 4.2 establishes (3).

4.9. Corollary to 3.4. Let $R \in \mathbf{M}_{k}^{\text {loc }}(Z)$ and $T \in \mathbf{M}_{k+m}^{\text {loc }}(X)$ be such that $L_{\mathscr{B}} \circ P_{\mathscr{B}} T=T$. Then:

(1) $\left\|L_{\mathscr{B}} R\right\|(f)=\int_{Z} \int_{Y_{z}} f d \mathcal{H}^{m} d\|R\| z$ for every $\left\|L_{\mathscr{B}} R\right\|$ integrable Borel function $f$ on $X$.

(2) $\pi_{\sharp}\|T\|=\left\|P_{\mathscr{B}} T\right\|\left\llcorner V_{\pi}\right.$. 
Proof. (1) is immediate, since $Z$ has the metric induced by $\pi$. For (2), use (1) and 4.1 to compute

$$
\begin{aligned}
\|T\|(\gamma \circ \pi) & =\int_{Z} \int_{Y_{z}} \gamma \circ \pi d \mathcal{F}^{m} d\left\|P_{\mathscr{B}} T\right\| z \\
& =\int_{Z} \gamma(z) \mathcal{H}^{m}\left(Y_{z}\right) d\left\|P_{\mathscr{B}} T\right\| z \\
& =\left\|P_{\mathscr{B}} T\right\|\left(\gamma V_{\pi}\right)
\end{aligned}
$$

whenever $\gamma \in \mathscr{Q}^{0}(Z)$.

5. Invariant solutions to the Plateau problem.

5.1. Let $b: T X \times T X \rightarrow \mathbf{R}$ denote the metric on $X$. If $\xi$ and $\eta$ are $C^{\infty}$ vectorfields, then $\nabla_{\xi} \eta$ will denote the covariant derivative of $\eta$ in the direction $\xi$. Let $\left\{\xi_{i}\right.$ : $i=1, \ldots, n\}$ be $C^{\infty}$ orthonormal vectorfields defined in an open set $W$ such that $\left\{\xi_{i}(x): i=1, \ldots, m\right\}$ spans $T_{x} Y_{z}$ for $x \in W$ and $z=\pi(x)$. Let $\omega^{i}$ be dual to $\xi_{i}$, $i=1, \ldots, n$,

$$
h_{i j}=b\left(\nabla_{\xi} \xi_{i}, \xi_{j}\right), \quad H_{j}=\sum_{i=1}^{m} h_{i j}
$$

and

$$
\kappa=\sum_{j=m+1}^{n} H_{j} \omega^{j}
$$

$\kappa$ is the mean curvature 1 -form of the fibres. Direct computations yield the equations (obtained in [L, p. 238] with opposite sign)

$$
d V=V \wedge \kappa, \quad d \Omega_{0}=\kappa \wedge \Omega_{0}+\Omega_{1},
$$

where $\left.\vec{Y}_{0}\right\lrcorner \Omega_{1}=0$, together with the fact that $\Omega_{1}=0$ if and only if the horizontal distribution of $\pi$ is involutive. (Details may be found in [B].) Since

$$
d \Omega=V^{-1} \wedge \Omega_{1},
$$

we infer that

$$
\partial \circ P_{\mathscr{B}}=P_{\mathscr{B}} \circ \partial
$$

if and only if the horizontal distribution of the submersion $\pi$ is involutive. We will assume for the remainder of the paper that this distribution is involutive.

5.2. TheOREM. Let $A \subset X$ be a $G$-invariant subset of $X$ and $B_{0} \in F_{k+m-1}^{l o c}(X)$ be such that spt $B_{0} \subset A, B_{0}=\partial T$ for some $T \in \mathbf{F}_{k+m}^{\text {loc }}(X) \cap \mathbf{M}_{k+m}^{\text {loc }}(X), L_{\mathscr{B}} \circ P_{\mathscr{B}}\left(B_{0}\right)$ $=B_{0}$ and $\mathcal{K}^{k+m}\left(\operatorname{spt} B_{0}\right)=0$. If $T_{0} \in \mathbf{F}_{k+m}^{\text {loc }}(X) \cap \mathbf{M}_{k+m}^{\text {loc }}(X)$ satisfies $\partial T_{0}=B_{0}$, spt $T_{0} \subset A$, and

$$
\mathbf{M}\left(T_{0}\right)=\inf \left\{\mathbf{M}(Q): Q \in \mathbf{F}_{k+m}^{\text {loc }}(X), \text { spt } Q \subset A, \partial Q=B_{0}\right\}<\infty,
$$

then $L_{\mathscr{B}} \circ P_{\mathscr{B}}\left(T_{0}\right)=T_{0}$.

Proof. Let $T_{2}=L_{\mathscr{B}} \circ P_{\mathscr{B}} T_{0}$. From 3.3(9), (7), (8) and 5.1 we have

$$
T_{2} \in \mathbf{F}_{k+m}^{\mathrm{loc}}(X), \quad \text { spt } T_{2} \subset A, \text { and } \partial T_{2}=B_{0} .
$$


Thus 4.8(3) and (1) imply

$$
\mathbf{M}\left(T_{2}\right) \leqslant \mathbf{M}\left(T_{0}\right) \leqslant \mathbf{M}\left(T_{2}\right)
$$

from which one obtains

$$
\vec{T}_{0}=(-1)^{m k}\left(\vec{T}_{0} \mathrm{~L} \Omega_{0}\right) \wedge \vec{Y}_{0}
$$

by 4.8(2). The theorem now follows from 3.6.

5.3. Remark. The variational problem considered in 5.2 is somewhat more general than the relative boundary problems treated by Federer [F2], because there are no assumptions on the boundary of $X$ (if that even makes sense). However, if $M$ is a compact manifold with boundary and $X=M \sim \partial M$, then the problems are the same. In fact we have the following: Let $C \subset A$ be compact subsets of $a$ Riemannian manifold $M$ with boundary. Denote $A^{\prime}=A \sim C$ and $M^{\prime}=M \sim C$ and suppose $B_{0} \in \mathbf{F}_{l}(M)$ is such that spt $B_{0} \subset A$. If either of the numbers

$$
\inf \left\{\mathbf{M}(T): T \in \mathbf{F}_{l}^{\text {loc }}\left(M^{\prime}\right), \operatorname{spt} T \subset A^{\prime}, \partial T=B_{0} \mid M^{\prime}\right\}
$$

or

$$
\inf \left\{\mathbf{M}(Q): Q \in \mathbf{F}_{l}(M), \text { spt } Q \subset A, \operatorname{spt}\left(\partial Q-B_{0}\right) \subset C\right\}
$$

is finite, then they are equal.

To verify this, note that if $Q$ is a candidate for the second infimum, then $Q \mid M^{\prime}$ is a candidate for the first with $\mathbf{M}\left(Q \mid M^{\prime}\right) \leqslant \mathbf{M}(Q)$. Conversely, if $T$ is a candidate for the first infimum and $\mathbf{M}(T)<\infty$, then for each $\varphi \in \mathcal{E}^{l}(M)$ we define $Q \in$ $\mathbf{F}_{l}(m)$ so that

$$
Q(\varphi)=\int_{M^{\prime}}\langle\vec{T}(x), \varphi(x)\rangle d\|T\| x
$$

and verify that $Q$ is a candidate for the first infimum with

$$
\mathbf{M}(Q)=\mathbf{M}(T) \text {. }
$$

5.4. As in [L, p. 236] we introduce the metric

$$
b_{k}=V_{\pi}^{2 / k} b_{\pi}
$$

on $Z$ for each $k=1, \ldots, n-m$. Each of these induces corresponding mass and comass norms which will be distinguished by the subscript $k$. Note that for $v$, $w \in \wedge_{k} T_{z}(Z)$

$$
b_{k}(v, w)=V_{\pi}^{2} b_{\pi}(v, w)
$$

and that since the norm \|\|$_{k}$ on $\bigwedge_{k} T_{u} B$ is equivalent to \|\|$, \mathbf{M}_{k}$ is equivalent to $\mathbf{M}$ for currents whose support is contained in a fixed compact set. Hausdorff measure in $Z$ will always be with respect to $b_{\pi}$.

5.5. Proposition. If $R \in \mathbf{M}_{k}^{\text {loc }}(Z)$, then

$$
\|R\|_{k}=\|R\| \mathrm{L} V_{\pi}=\pi_{\sharp}\left\|L_{\mathscr{B}} R\right\| .
$$

Consequently, if $T \in \mathbf{M}_{k}^{\text {loc }}(X)$ with $L_{\mathscr{B}} \circ P_{\mathscr{B}}(T)=T$ then

$$
\mathbf{M}_{k}\left(P_{\mathfrak{B}} T\right)=\mathbf{M}(T) \text {. }
$$


Proof. If $R \in \mathbf{M}_{k}^{\text {loc }}(Z)$ and $f$ is nonnegative and continuous, we have

$$
\begin{aligned}
\|R\|_{k}(f) & =\sup \left\{R(\varphi): \varphi \in \mathscr{D}^{k}(Z),\|\varphi\|_{k} \leqslant f\right\} \\
& =\sup \left\{R(\varphi): \varphi \in \mathscr{D}^{k}(Z), V_{\pi}^{-1}\|\varphi\| \leqslant f\right\} \\
& =\|R\|\left(f V_{\pi}\right) .
\end{aligned}
$$

The result now follows from 4.9(2) and 3.3(5).

5.6. THEOREM. Let $A \subset X$ be $G$-invariant and $B_{0} \in \mathbf{F}_{m+k-1}(X)$ be such that spt $B_{0} \subset A, B_{0}=\partial T$ for some $T \in \mathbf{F}_{k+m}^{\text {loc }}(X) \cap \mathbf{M}_{k+m}^{\text {loc }}(X)$ and $L_{\mathscr{B}} \circ P_{\mathscr{B}}\left(B_{0}\right)=B_{0}$. If

$$
\begin{aligned}
& \mu_{1}=\inf \left\{\mathbf{M}(T): T \in \mathbf{F}_{k+m}^{\text {loc }}(X), \text { spt } T \subset A, \partial T=B_{0}\right\}, \\
& \mu_{2}=\inf \left\{\mathbf{M}_{k}(R): R \in \mathbf{F}_{k}^{\text {loc }}(Z), \operatorname{spt} R \subset \pi(A), \partial R=P_{\mathscr{B}_{B}} B_{0}\right\},
\end{aligned}
$$

then $\mu_{1}=\mu_{2}$.

Proof. For $T \in \mathbf{F}_{k+m}^{\text {loc }}(X)$ such that $\mathbf{M}(T)<\infty$, spt $T \subset A$ and $\partial T=B_{0}$, one uses 3.3(9), 3.1, [F1, 4.1.7] and 5.1 to conclude $P_{\mathscr{B}} T \in \mathbf{F}_{k}^{\mathrm{loc}}(Z) \cap \mathbf{M}_{k}^{\mathrm{loc}}(Z)$, spt $P_{\mathscr{B}} T$ $\subset \pi(A)$ and $\partial P_{\mathscr{B}} T=P_{\mathscr{B}} B_{0}$. Application of 3.3(5), 5.5 and 4.8(1), (3) yields

$$
\mathbf{M}_{k}\left(P_{\mathscr{B}} T\right)=\mathbf{M}\left(L_{\mathscr{B}} \circ P_{\mathscr{B}} T\right) \leqslant \mathbf{M}(T) \text {. }
$$

Hence $\mu_{2} \leqslant \mu_{1}$.

Now suppose $R \in \mathbf{F}_{k}^{\text {loc }}(Z)$ satisfies $\mathbf{M}(R)<\infty$, spt $R \subset \pi(A)$ and $\partial R=P_{\mathscr{B}} B_{0}$. By virtue of 5.5, 3.3(9), (7), (5), (8) and 5.1, one has

$$
\begin{gathered}
\mathbf{M}_{k}(R)=\mathbf{M}\left(L_{\mathscr{B}} R\right), \\
L_{\mathscr{B}} R \in \mathbf{F}_{k+m}^{\mathrm{loc}}(X) \cap \mathbf{M}_{k+m}^{\mathrm{loc}}(X), \\
\operatorname{spt} L_{\mathscr{B}} R \subset A,
\end{gathered}
$$

and

$$
\partial L_{\mathscr{B}} R=B_{0}
$$

and thus $\mu_{1} \leqslant \mu_{2}$.

The theorem now follows if either infimum is finite and, of course, is obvious otherwise.

5.7. THEOREM. Let $A$ and $C$ be compact $G$-invariant sets which are $G$-invariant Lipschitz neighborhood retracts with $C \subset A$. Let $B_{0} \in \mathscr{F}_{m}(X)$ be such that $\mathcal{H}^{m+1}\left(\operatorname{spt} B_{0}\right)=0$ and there exists $T_{1} \in \mathscr{R}_{m+1, A}(X)$ such that $\operatorname{spt}\left(\partial T_{1}-B_{0}\right) \subset C$ and $g_{\sharp} T_{1}=T_{1}$ for all $g \in G$. Then there exists $T_{0} \in \Re_{m+1, A}(X)$ such that $\operatorname{spt}\left(\partial T_{0}-B_{0}\right) \subset C, g_{\sharp} T_{0}=T_{0}$ for all $g \in G$ and

$$
\mathbf{M}\left(T_{0}\right)=\mu_{0}=\inf \left\{\mathbf{M}(Q): Q \in \mathcal{R}_{m+1, A}(X), \operatorname{spt}\left(\partial Q-B_{0}\right) \subset C\right\} .
$$

Moreover, if $T \in \mathcal{R}_{m+1, A}(X)$ is such that $\operatorname{spt}\left(\partial T-B_{0}\right) \subset C$ and $\mathbf{M}(T)=\mu_{0}$, then $L_{\mathscr{B}} \circ P_{\mathscr{B}} T=T$ (hence $g_{\sharp} T=T$ for $g \in G$ ).

Proof. Let

$$
\mu_{1}=\inf \left\{\mathbf{M}(T): T \in \mathbf{F}_{m+1, A}(X), \operatorname{spt}\left(\partial T-B_{0}\right) \subset C\right\}
$$


and

$$
\mu_{2}=\inf \left\{\mathbf{M}(T): T \in \mathscr{R}_{m+1, A}(X), \operatorname{spt}\left(\partial T-B_{0}\right) \subset C, L_{\mathscr{B}} \circ P_{\mathscr{B}} T=T\right\} .
$$

Clearly $\mu_{1} \leqslant \mu_{0} \leqslant \mu_{2}$.

Let $X^{\prime}=X \sim C, A^{\prime}=A \sim C, Z^{\prime}=Z \sim \pi(C)$ and $B_{0}^{\prime}=B_{0} \mid X$. Arguing as in 5.3 it is an easy matter to verify that

$$
\mu_{1}=\inf \left\{\mathbf{M}(Q): Q \in \mathbf{F}_{m+1}^{\text {loc }}\left(X^{\prime}\right), \text { spt } Q \subset A^{\prime}, \partial Q=B_{0}^{\prime}\right\} .
$$

An application of 5.6 yields the equality

$$
\mu_{1}=\inf \left\{\mathbf{M}_{1}(F): F \in \mathbf{F}_{1}^{\mathrm{loc}}\left(Z^{\prime}\right), \text { spt } F \subset \pi\left(A^{\prime}\right), \partial F=P_{\mathscr{B}} B_{0}^{\prime}\right\}
$$

from which

$$
\mu_{1}=\inf \left\{\mathbf{M}_{1}(R): R \in \mathbf{F}_{1}(Z) \cap \mathbf{M}_{1}(Z), \operatorname{spt} R \subset \pi(A), \operatorname{spt}\left(\partial R-P_{Q_{B}} B_{0}\right) \subset \pi(C)\right\}
$$

follows using the compactness of $\pi(A)$.

For $G$-invariant $T_{1} \in \mathcal{R}_{m+1, A}(X)$ one can use [F1, 4.1.28(5)], 4.3, 4.2, 4.9(2), 4.1 and 3.3(4) to show that $P_{\mathscr{B}} T_{1} \in \Re_{1, \pi(A)}(Z)$. Now $b_{\pi}$-isometrically embed $Z$ as a proper submanifold of $\mathbf{R}^{N}$ for some $N$, use [F1, p. 373 and 3.1.20] in order to apply [F2, 5.12] with $T=P_{\mathscr{B}} T_{1}$ and [F1, 4.2.16(3)], and conclude

$$
\mu_{1}=\inf \left\{\mathbf{M}_{1}(R): R \in \mathcal{R}_{1, \pi(A)}(Z), \operatorname{spt}\left(\partial R-P_{\mathscr{B}} B_{0}\right) \subset \pi(C)\right\} .
$$

For $R \in \mathcal{R}_{1, \pi(A)}(Z)$ such that $\operatorname{spt}\left(\partial R-P_{\mathscr{B}} B_{0}\right) \subset \pi(C)$ use [BJ1, 3.5(6)], 3.2 and $3.3(7),(8),(5)$ to obtain

$$
L_{\mathscr{B}} R \in \mathcal{R}_{m+1, A}(Z), \quad \operatorname{spt}\left(\partial L_{\mathscr{B}} R-B_{0}\right) \subset C
$$

and

$$
L_{\mathscr{B}} \circ P_{\mathscr{B}}\left(L_{\mathscr{B}} R\right)=L_{\mathscr{B}} R
$$

Hence 5.5 implies

$$
\mu_{2} \leqslant \mu_{1}
$$

Now suppose $T_{0} \in \mathscr{R}_{m+1, A}(X)$ satisfies $\operatorname{spt}\left(\partial T_{0}-B_{0}\right) \subset C$ and $\mathbf{M}\left(T_{0}\right)=\mu_{0}$. (Existence of such a $T_{0}$ is guaranteed by [F1, 5.1.6(1)].) Then

$$
\mu_{1} \leqslant \mathbf{M}\left(L_{\mathfrak{B}} \circ P_{\mathfrak{B}}\left(T_{0}\right)\right) \leqslant \mathbf{M}\left(T_{0}\right)=\mu_{0}=\mu_{1}
$$

and so by 5.2 ,

$$
L_{\mathfrak{B}} \circ P_{\mathscr{B}}\left(T_{0}\right)=T_{0} .
$$

Finally, invariance of $T_{0}$ follows from 4.2.

5.8. REMARK. In view of $[F 1,4.4 .1]$, whenever $P_{\mathscr{B}} B_{0} \in \mathscr{F}_{0}(Z)$ and $A$ is connected one can replace the hypotheses concerning the existence of $T_{1}$ in 5.7 by $L_{\mathscr{B}} \circ P_{\mathscr{B}} B_{0}$ $=B_{0}$, if $C \neq \varnothing$, or by $L_{\mathscr{B}} \circ P_{\mathscr{B}} B_{0}=B_{0}$ and $B_{0}(\Omega)=0$, if $C=\varnothing$.

5.9. Suppose $G \subset \mathrm{SO}(n)$ is a connected closed Lie subgroup acting orthogonally on $\mathbf{R}^{n}$. Assume there are no exceptional orbits. Let $X$ be the set of points in $\mathbf{R}^{n}$ which lie on principal orbits and $S=\mathbf{R}^{n} \sim x$. By [BG, IV.3.1], $X$ is an open dense subset of $\mathbf{R}^{n}$ and by [BG, IV.3.1 and 4.4], $Z=X / G$ is orientable. So if we further assume that the distribution of $(n-m)$-planes orthogonal to the principal orbits is involutive, then all of the previous results apply to $X$. 
5.10. TheOREM. Let $B_{0} \in \mathbf{I}_{m}\left(\mathbf{R}^{n}\right)$ be such that $g_{\sharp} B_{0}=B_{0}$ for all $g \in G, \partial B_{0}=0$ and spt $B_{0} \subset X$. Then for every $T_{0} \in \mathbf{I}_{m+1}\left(\mathbf{R}^{n}\right)$ such that $\partial T_{0}=B_{0}$ and

$$
\mathbf{M}\left(T_{0}\right)=\inf \left\{\mathbf{M}(T): T \in \mathbf{I}_{m+1}\left(\mathbf{R}^{n}\right), \partial T=B_{0}\right\}
$$

one has

$$
g_{\sharp} T_{0}=T_{0} \quad \text { for all } g \in G .
$$

Proof. Find $r$ so that spt $B_{0} \subset \mathrm{U}(0, r)$ and let $K=\mathbf{B}(0,2 r)$ and $K^{\prime}=K \cap X$. Let

$$
\begin{aligned}
& \Gamma_{1}=\mathbf{F}_{m+1, K}\left(\mathbf{R}^{n}\right) \cap\left\{T: \operatorname{spt}\left(\partial T-B_{0}\right) \subset S\right\}, \\
& \Gamma_{2}=\mathbf{F}_{m+1}^{\text {loc }}(X) \cap\left\{Q: \text { spt } Q \subset K^{\prime}, \partial Q=B_{0}\right\}, \\
& \Gamma_{3}=\mathbf{F}_{1}^{\text {loc }}(Z) \cap\left\{F: \text { spt } F \subset \pi\left(K^{\prime}\right), \partial F=P_{\mathscr{B}} B_{0}\right\}, \\
& \Gamma_{4}=\mathcal{R}_{1}^{\text {loc }}(Z) \cap\left\{R: \text { spt } R \subset \pi\left(K^{\prime}\right), \partial R=P_{\mathscr{B}} B_{0}\right\}, \\
& \Gamma_{5}=\mathcal{R}_{m+1}^{\text {loc }}(X) \cap\left\{Q: \operatorname{spt} Q \subset K^{\prime}, \partial Q=B_{0}, L_{\mathscr{B}} \circ P_{\mathscr{B}} Q=Q\right\}, \\
& \Gamma_{6}=\mathcal{R}_{m+1, K}\left(\mathbf{R}^{n}\right) \cap\left\{T: \operatorname{spt}\left(\partial T-B_{0}\right) \subset S, g_{\sharp} T=T \text { for all } g \in G\right\}, \\
& \Gamma_{7}=\mathbf{I}_{m+1, K}\left(\mathbf{R}^{n}\right) \cap\left\{T: \partial T=B_{0}, g_{\sharp} T=T \text { for all } g \in G\right\}, \\
& \Gamma_{8}=\mathbf{I}_{m+1}\left(\mathbf{R}^{n}\right) \cap\left\{T: \partial T=B_{0}\right\},
\end{aligned}
$$

and $\mu_{i}=\inf \left\{\Psi(T): T \in \Gamma_{i}\right\}, i=1, \ldots, 8$, where $\Psi=\mathbf{M}$ for $i \notin\{3,4\}$ and $\Psi=\mathbf{M}_{1}$ for $i \in\{3,4\}$.

The theorem will be proved by verifying the followng five statements:

1. $\mu_{1} \leqslant \mu_{8} \leqslant \mu_{7}$,

2. $\mu_{3}=\mu_{2} \leqslant \mu_{1}$,

3. $\mu_{7} \leqslant \mu_{6} \leqslant \mu_{5} \leqslant \mu_{4}$,

4. $\mu_{4} \leqslant \mu_{3}$,

5. $g_{\sharp} T_{0}=T_{0}$ for all $g \in G$.

Part 1. The inequalities

$$
\mu_{1} \leqslant \inf \left\{\mathbf{M}(T): T \in \mathbf{I}_{m+1, K}\left(\mathbf{R}^{n}\right), \partial T=B_{0}\right\}
$$

and

$$
\mu_{8} \leqslant \mu_{7}
$$

are obvious. The desired result now follows from [BJ3, 3.8].

Part 2. If $T \in \Gamma_{1}$, then $Q=T \mid X \in \Gamma_{2}$ with $\mathbf{M}(Q) \leqslant \mathbf{M}(T)$. Hence $\mu_{1} \geqslant \mu_{2}$. The equality $\mu_{2}=\mu_{3}$ is a consequence of 5.6.

Part 3. To see that $\mu_{4} \geqslant \mu_{5}$, take $R \in \Gamma_{4}$ and use [BJ1, 3.5(6)], 3.3(7), (8), (5) and 5.5 to conclude $L_{\mathscr{B}} R \in \Gamma_{5}$ with $\mathbf{M}\left(L_{\mathscr{B}} R\right)=\mathbf{M}_{1}(R)$.

For $Q \in \Gamma_{5}$ such that $\mathbf{M}(Q)<\infty$, define $T \in \mathcal{R}_{m+1}\left(\mathbf{R}^{n}\right)$ by

$$
T(\varphi)=\int_{X}\langle\vec{Q}, \varphi\rangle d\|Q\|
$$

whenever $\varphi \in \mathcal{E}^{m+1}\left(\mathbf{R}^{n}\right)$. It is an easy matter to check that $T \in \Gamma_{6}$ and $\mathbf{M}(T)=$ $\mathbf{M}(Q)$. Hence $\mu_{6} \leqslant \mu_{5}$.

The existence of a current $T \in \Gamma_{6}$ such that $\mathbf{M}(T)=\mu_{6}$ can be proved as in [F1, 5.1.6]. In fact, using the notation of [F1, 5.1.6], $H(r)$ is $G$-invariant so that $Q_{i} L H(r)$ 
is $G$-invariant for $\mathcal{L}^{1}$ almost every $r$. That

$$
\liminf _{i \rightarrow \infty} \mathcal{F}_{K}\left(\left(Q_{i}-S\right)\llcorner H(r))=0\right.
$$

implies that a subsequence of $\left\{Q_{i}\llcorner H(r)\}\right.$ converges weakly to $S\llcorner H(r)$. Hence $S\llcorner H(r)$ is $G$-invariant for arbitrarily small $r$ from which the invariance of $S$ follows. One now applies $[\mathbf{L}, 4.4]$ to obtain $\mu_{7} \leqslant \mu_{6}$.

Part 4. Let $\varepsilon_{0}=\operatorname{dist}\left(\operatorname{spt} B_{0}, S\right)$. For $\varepsilon \in\left(0, \varepsilon_{0}\right)$, denote

$$
Z_{\varepsilon}=\pi(\text { int } K \cap\{x: \operatorname{dist}(x, S)>\varepsilon\}), \quad C_{\varepsilon}=\operatorname{Clos}\left(Z_{\varepsilon}\right) \sim Z_{\varepsilon},
$$

and

$$
\mu_{4}(\varepsilon)=\inf \left\{M_{1}(R): R \in \mathscr{R}_{1}(Z), \operatorname{spt} R \subset \operatorname{Clos} Z_{\varepsilon}, \operatorname{spt}\left(\partial R-P_{\mathscr{B}_{B}} B_{0}\right) \subset C_{\varepsilon}\right\} .
$$

We begin by showing

$$
\mu_{4} \leqslant \lim _{\varepsilon \rightarrow 0^{+}} \mu_{4}(\varepsilon) \text {. }
$$

Fix $\varepsilon \in\left(0, \varepsilon_{0}\right)$ and $b_{\pi}$-isometrically embed $Z$ into some Euclidean space. By applying [F1, 3.1.20, p. 373 and 5.1.6], one finds $R_{e} \in R_{1}(Z)$ such that spt $R_{e} \subset$ $\pi\left(\operatorname{Clos} Z_{\varepsilon}\right)$, spt $\partial R_{\varepsilon}-P_{\mathscr{B}} B_{0} \subset C_{\varepsilon}$ and $\mathbf{M}_{1}\left(R_{\varepsilon}\right)=\mu_{4}(\varepsilon)$.

One can apply [F1, 4.2.1 and 4.1.17] to obtain for $\mathcal{L}^{1}$ a.e. $\delta \in\left[0, \varepsilon_{0}-\varepsilon\right)$

$$
\partial\left(R_{\varepsilon}\left\llcorner Z_{\varepsilon+\delta}\right) \varphi=-\left\langle R_{\varepsilon}, \operatorname{dist}(\cdot, S), \varepsilon+\delta\right\rangle \varphi\right.
$$

whenever $\varphi \in \mathcal{E}^{0}(Z)$ and spt $\partial R_{\varepsilon} \cap \operatorname{spt} \varphi=\varnothing$. Then

$$
R_{\varepsilon, \delta}=R_{\varepsilon}\left\llcorner Z_{\varepsilon+\delta} \in I_{1}(Z)\right.
$$

follows from [F1, 4.3.2(2) and 4.2.16(2)] for $\mathcal{L}^{1}$ a.e. $\delta \in\left[0, \varepsilon_{0}-\varepsilon\right)$. Suppose $R_{\varepsilon, \delta} \in$ $I_{1}(Z)$. We now use [F1, 4.2.25] and the fact that $\operatorname{spt}\left(\Sigma T_{i}\right)=\cup \operatorname{spt} T_{i}$ whenever $\mathbf{N}\left(\Sigma T_{i}\right)=\sum \mathrm{N}\left(T_{i}\right)$ to find indecomposable $R_{\varepsilon, \delta}^{j} \in \mathbf{I}_{1}(Z)$ such that

$$
R_{\varepsilon, \delta}=\sum_{i=1}^{\infty} R_{\varepsilon, \delta}^{i} \quad \text { and } \quad \mathrm{N}\left(R_{\varepsilon, \delta}\right)=\sum_{j=1}^{\infty} \mathrm{N}\left(R_{\varepsilon, \delta}^{j}\right)
$$

where $R_{\varepsilon, \delta}^{j}$ is an oriented simple rectifiable curve for each $j$. Then

$$
\mathbf{M}_{1}\left(R_{\varepsilon, \delta}\right)=\sum_{j=1}^{\infty} \mathbf{M}_{1}\left(R_{\varepsilon, \delta}^{j}\right)
$$

Let

$$
J_{i}=\left\{j: \mathbf{M}\left(\partial R_{\varepsilon, \delta}^{j}\left\llcorner Z_{\varepsilon+\delta}\right)=i\right\}, \quad i=0,1,2 .\right.
$$

For each $j \in J_{1}$, let $q_{j} \in Z$ and $\sigma_{j} \in\{1,-1\}$ be such that

$$
\partial R_{\varepsilon, \delta}^{j}\left\llcorner C_{\varepsilon+\delta}=\sigma_{j} \delta_{q_{j}} .\right.
$$

Note that $\operatorname{dist}\left(\pi^{-1}\left(q_{j}\right), S\right)=\varepsilon+\delta$.

Choose $x_{j} \in \pi^{-1}\left\{q_{j}\right\}$ and find $y_{j} \in S$ such that

$$
\left|x_{j}-y_{j}\right|=\operatorname{dist}(x, S)=\varepsilon+\delta \text {. }
$$

Let $\gamma_{j}:[0,1) \rightarrow X$ be defined by the formula

$$
\gamma_{j}(t)=(1-t) x_{j}+t y_{j}
$$


and let $L_{j}=\sigma_{j}(\pi \circ \gamma)_{\sharp}[0,1) \in \mathbf{I}_{1}^{\text {loc }}(Z)$ and $V_{0}=\sup \left\{V(x): x \in K^{\prime}\right\}$. Then

$$
\mathbf{M}_{1}\left(L_{j}\right) \leqslant V_{0}(\varepsilon+\delta) \text {. }
$$

If we let

$$
R=\sum_{j \in J_{1}}\left(R_{\varepsilon, \delta}^{j}+L_{j}\right)+\sum_{j \in J_{2}} R_{\varepsilon, \delta}^{j}
$$

then we have $R \in \mathcal{R}_{1}^{\text {loc }}(Z)$, spt $R \subset \pi\left(K^{\prime}\right)$, and

$$
\begin{aligned}
\partial R & =\sum_{j \in J_{1}} \partial R_{\varepsilon, \delta}^{j}-\sigma_{j} \delta_{q_{j}}+\sum_{j \in J_{2}} \partial R_{\varepsilon, \delta}^{j} \\
& =\sum_{j \in J_{1}} \partial R_{\varepsilon, \delta}^{j}\left\llcorner Z_{\varepsilon+\delta}+\sum_{j \in J_{2}} \partial R_{\varepsilon, \delta}^{j}\right. \\
& =\left(\partial R_{\varepsilon, \delta}\right)\left\llcorner Z_{\varepsilon+\delta}=P_{\mathscr{B}} B_{0} .\right.
\end{aligned}
$$

Hence

$$
\begin{aligned}
\mu_{4} & \leqslant \mathbf{M}_{1}(R) \leqslant V_{0}\left(\operatorname{card} J_{1}\right)(\varepsilon+\delta)+\sum_{j \in J_{1} \cup J_{2}} \mathbf{M}_{1}\left(R_{e, \delta}^{j}\right) \\
& \leqslant V_{0} \mathbf{M}\left(P_{\mathscr{B}} B_{0}\right)(\varepsilon+\delta)+\mathbf{M}_{1}\left(R_{\varepsilon, \delta}\right) \\
& \leqslant V_{0} \mathbf{M}\left(P_{\mathscr{B}} B_{0}\right)(\varepsilon+\delta)+\mathbf{M}_{1}\left(R_{\varepsilon}\right) \\
& =V_{0} \mathbf{M}\left(P_{\mathscr{B}} B_{0}\right)(\varepsilon+\delta)+\mu_{4}(\varepsilon) .
\end{aligned}
$$

Thus $\mu_{4} \leqslant \lim _{\varepsilon \rightarrow 0} \mu_{4}(\varepsilon)$.

For $\varepsilon>0$ we can find an open set $V \subset Z$ such that Clos $V$ is compact, $V \supset Z_{\varepsilon}$ and Bdry $V$ is a $C^{\infty}$ submanifold of $Z$. Then by restriction and [F2, 5.12] we have

$$
\begin{aligned}
\mu_{3} & \geqslant \inf \left\{\mathbf{M}_{1}(F): F \in \mathbf{F}_{1}(Z), \operatorname{spt} F \subset \operatorname{Clos} V, \operatorname{spt}\left(\partial F-P_{\mathscr{B}^{B}} B_{0}\right) \subset \operatorname{Bdry} V\right\} \\
& =\inf \left\{\mathbf{M}_{1}(R): R \in \mathcal{R}_{1}(Z), \operatorname{spt} R \subset \operatorname{Clos} V, \operatorname{spt}\left(\partial R-P_{\mathscr{B}} B_{0}\right) \subset \operatorname{Bdry} V\right\} \\
& \geqslant \mu_{4}(\varepsilon) .
\end{aligned}
$$

Part 5. Assume $T_{0} \in \mathbf{I}_{m+1}\left(\mathbf{R}^{n}\right)$ satisfies $\partial T_{0}=B_{0}$ and $\mathbf{M}\left(T_{0}\right)=\mu_{8}$. The inequalities of the first four parts show that $\mu_{i}=\mu_{j}$ for $i, j=1, \ldots, 8$. In particular, 5.2, 4.2 and $\mu_{2}=\mu_{8}$ imply that $T_{0} \mid X$ is $G$-invariant. Hence $T_{0} L X$ is $G$-invariant and we may use the equality $\mu_{1}=\mu_{8}$ to see that $T_{0}=T_{0}\llcorner X$.

5.11. Corollary. If $B_{0} \in \mathbf{I}_{m}\left(\mathbf{R}^{n}\right)$ satisfies $g_{\sharp} B_{0}=B_{0}$ for all $g \in G, \partial B_{0}=0$ and spt $B_{0} \subset X$, then $P_{\mathscr{B}}$ is a bijection from

$$
\mathbf{I}_{m+1}\left(\mathbf{R}^{n}\right) \cap\left\{T: \partial T=B_{0}, T \text { is absolutely area minimizing }\right\}
$$

to

$\Re_{1}^{\text {loc }}(Z) \cap\left\{R: \partial R=P_{\mathscr{B}} B_{0}, \mathbf{M}_{1}(R)=\inf \left\{\mathbf{M}_{1}\left(R^{\prime}\right): R^{\prime} \in \Re_{1}^{\text {loc }}(Z), \partial R^{\prime}=P_{\mathscr{B}} B_{0}\right\}\right\}$.

\section{REFERENCES}

[B] D. E. Bindschadler, Invariant and singular solutions to the Plateau problem in Riemannian manifolds, Ph.D. Thesis, Indiana University, 1976.

[BG] G. E. Bredon, Introduction to compact transformation groups, Academic Press, New York, 1972.

[BJ1] J. E. Brothers, Integral geometry in homogeneous spaces, Trans. Amer. Math. Soc. 124 (1966), 480-517. 
[BJ2] __ A characterization of integral currents, Trans. Amer. Math. Soc. 150 (1970), 301-325.

[BJ3] _ The structure of solutions to Plateau's problem in the n-sphere, J. Differential Geometry 11 (1976), 387-400.

[F1] H. Federer, Geometric measure theory, Springer-Verlag, Berlin and New York, 1969.

[F2] __, Real flat chains, cochains and variational problems, Indiana Univ. Math. J. 24 (1974), 351-407.

[FF] H. Federer and W. H. Fleming, Normal and integral currents, Ann. of Math. (2) 72 (1960), 458-520.

[L] H. B. Lawson, The equivariant Plateau problem and interior regularity, Trans. Amer. Math. Soc. 173 (1972), 231-249.

Department of Mathematics, Wayne State University, Detroit, Michigan 48202 